\title{
Probing $d^{8}-d^{8}$ Interactions in Luminescent Mono- and Binuclear Cyclometalated Platinum(II) Complexes of 6-Phenyl-2,2'-bipyridines
}

\author{
Siu-Wai Lai, ${ }^{\dagger}$ Michael Chi-Wang Chan, ${ }^{\dagger}$ Tsz-Chun Cheung, ${ }^{\dagger}$ Shie-Ming Peng, ${ }^{\ddagger}$ and \\ Chi-Ming Che ${ }^{*, \dagger}$
}

Department of Chemistry, The University of Hong Kong, Pokfulam Road, Hong Kong, and Department of Chemistry, National Taiwan University, Taipei, Taiwan

Received February 26, 1999

\begin{abstract}
A series of luminescent mono- and binuclear cyclometalated platinum(II) complexes, namely $\left[\mathrm{Pt}\left(\mathrm{L}^{1-6}\right) \mathrm{Cl}\right](\mathbf{1 a}-$ 6a; $\mathrm{HL}^{1-6}=$ 4-(aryl)-6-phenyl-2,2'-bipyridine; aryl = H (1), phenyl (2), 4-chlorophenyl (3), 4-tolyl (4), 4-methoxyphenyl (5), 3,4,5-trimethoxyphenyl (6)), $\left[\mathrm{Pt}\left(\mathrm{L}^{1}\right) \mathrm{E}\right]^{+}\left(\mathrm{E}=\right.$ py $\left.(\mathbf{7}), \mathrm{PPh}_{3}(\mathbf{8})\right),\left[\mathrm{Pt}_{2}\left(\mathrm{~L}^{1-6}\right)_{2}\left(\mu\right.\right.$-dppm) ${ }^{2+}$ $\left(\mathbf{1 b}-\mathbf{6 b}, \mathrm{dppm}=\right.$ bis(diphenylphosphino)methane), $\left[\mathrm{Pt}_{2}\left(\mathrm{~L}^{1}\right)_{2}(\mu-\mathrm{pz})\right]^{+}(\mathbf{9}, \mathrm{Hpz}=$ pyrazole $)$, and $\left[\mathrm{Pt}_{2}\left(\mathrm{~L}^{1}\right)_{2}\left(\mu-\mathrm{dppC}_{n}\right)\right]^{2+}$ $\left(\operatorname{dppC}_{n}=\right.$ bis(diphenylphosphino)propane $(\mathbf{1 0}, n=3)$ and -pentane $\left.(\mathbf{1 1}, n=5)\right)$, were synthesized in order to examine fluid- and solid-state oligomeric $\mathrm{d}^{8}-\mathrm{d}^{8}$ and ligand-ligand interactions. The molecular structures of $\mathbf{4 b}\left(\mathrm{ClO}_{4}\right)_{2}$ and $\mathbf{9}\left(\mathrm{PF}_{6}\right)$ reveal intramolecular $\mathrm{Pt}-\mathrm{Pt}$ distances of 3.245(1) and 3.612(2) $\AA$, respectively. While minimal metal-metal communication is expected for $\mathbf{9}$, weak $\pi-\pi$ interactions are possible. All complexes described in this work are emissive in fluid solution at room temperature. Negligible changes in emission energy are detected by incorporating different aryl substituents into the 4-position of 6-phenyl-2,2'-bipyridine, and this indicates little electronic delocalization between them. Self-quenching of the ${ }^{3} \mathrm{MLCT}$ emission by the mononuclear derivatives are observed in $\mathrm{CH}_{2} \mathrm{Cl}_{2}$ at $298 \mathrm{~K}$, and a red shift in the emission energy is exhibited by complex 7 in acetonitrile at $77 \mathrm{~K}$. The fluid emissions of the $\mu$-dppm species $\mathbf{1 b}-\mathbf{6 b}$ at $\lambda_{\max } 652-662 \mathrm{~nm}$ appear at substantially lower energies than their mononuclear counterparts and show dramatic solvatochromic effects. These emissions are ascribed to ${ }^{3}\left[\mathrm{~d} \sigma^{*}, \pi^{*}\right]$ excited states. In contrast, the emission of $\mathbf{1 0}$ and $\mathbf{1 1}$, bearing long bridging diphosphine ligands, are attributed to ${ }^{3} \mathrm{MLCT}$ states of non-interacting $\left[\mathrm{Pt}\left(\mathrm{L}^{1}\right)\right]$ moieties. Significantly, the luminescence of the $\mu$-pyrazolate complex 9 displays transitional features which are reminiscent of both ${ }^{3}\left[\mathrm{~d} \sigma^{*}, \pi^{*}\right]$ and ${ }^{3}$ MLCT excited states. Hence a relationship is observed between emission energy, the nature of the lowest energy excited state, and metal-metal interactions. The excited-state redox potential $\left[E\left(* \mathrm{Pt}_{2}{ }^{2+} / \mathrm{Pt}_{2}{ }^{+}\right)\right]$of $\mathbf{1 b}$ has been estimated by electrochemical studies (1.61 V vs NHE) and by quenching experiments with aromatic hydrocarbons $(1.63 \mathrm{~V}$ vs NHE).
\end{abstract}

\section{Introduction}

Luminescent coordinatively unsaturated metal complexes are appealing from a photochemical perspective. While saturated congeners such as $\left[\mathrm{Ru}(\mathrm{bpy})_{3}\right]^{2+}$ (bpy $=2,2^{\prime}$-bipyridine) are restricted to outer-sphere interactions with substrates, these chromophores allow inner-sphere electron-transfer reactions, and applications for chemical sensing, ${ }^{1-6}$ solar energy conversion, and photocatalysis ${ }^{7-9}$ have been developed. Investigations into square planar $\mathrm{d}^{8}$ platinum(II) compounds have been prominent

* Corresponding author. Fax: (852) 2857 1586. E-mail: cmche@hkucc. hku.hk.

$\doteqdot$ The University of Hong Kong.

$\doteqdot$ National Taiwan University.

(1) Kunugi, Y.; Mann, K. R.; Miller, L. L.; Exstrom, C. L. J. Am. Chem. Soc. 1998, 120, 589 .

(2) Mansour, M. A.; Connick, W. B.; Lachicotte, R. J.; Gysling, H. J.; Eisenberg, R. J. Am. Chem. Soc. 1998, 120, 1329.

(3) (a) Wu, L. Z.; Cheung, T. C.; Che, C. M.; Cheung, K. K.; Lam, M. H. W. Chem. Commun. 1998, 1127. (b) Liu, H. Q.; Cheung, T. C.; Che, C. M. Chem. Commun. 1996, 1039. (c) Liu, H. Q.; Peng, S. M.; Che, C. M. J. Chem. Soc., Chem. Commun. 1995, 509. (d) Wong, K. H.; Chan, M. C. W.; Che, C. M. Chem. Eur. J. in press.

(4) Peyratout, C. S.; Aldridge, T. K.; Crites, D. K.; McMillin, D. R. Inorg. Chem. 1995, 34, 4484.

(5) Lee, W. W. S.; Wong, K. Y.; Li, X. M. Anal. Chem. 1993, 65, 255.

(6) (a) Vickery, J. C.; Olmstead, M. M.; Fung, E. Y.; Balch, A. L. Angew. Chem., Int. Ed. Engl. 1997, 36, 1179. (b) Gade, L. H. Angew. Chem., Int. Ed. Engl. 1997, 36, 1171. since this class of molecules can mediate excited-state atom transfer reactions and bond activation. In particular, the prolific excited-state chemistry of the binuclear derivative $\left[\mathrm{Pt}_{2}(\mu\right.$ $\left.\left.\mathrm{P}_{2} \mathrm{O}_{5} \mathrm{H}_{2}\right)_{4}\right]^{4-}$ has been demonstrated. ${ }^{10}$ The triplet $\left(\mathrm{d} \sigma^{*}, \mathrm{p} \sigma\right)$ excited state, which is a manifestation of the $\mathrm{d}^{8}-\mathrm{d}^{8}$ interaction between the diplatinum centers, is capable of $\mathrm{C}-\mathrm{H}$ and $\mathrm{C}$-halogen bond cleavage and electron-transfer reactions.

The propensity for square planar $\mathrm{d}^{8}$ complexes to engage in metal-metal interactions and form extended linear-chain solidstate structures has been extensively studied. ${ }^{11}$ Unusual colors and strong emission, as well as highly anisotropic properties, are often the result of such stacking interactions. Platinum(II)

(7) (a) Gray, H. B.; Maverick, A. W. Science 1981, 214, 1201. (b) Nocera, D. G.; Maverick, A. W.; Winkler, J. R.; Che, C. M.; Gray, H. B. In ACS Symposium Series, Vol. 211; Chisholm, M. H., Ed.; American Chemical Society: Washington, DC, 1982; p 21.

(8) Nocera, D. G. Acc. Chem. Res. 1995, 28, 209.

(9) Roundhill, D. M. Photochemistry and Photophysics of Metal Complexes; Plenum Press: New York, 1994; p 356.

(10) Roundhill, D. M.; Gray, H. B.; Che, C. M. Acc. Chem. Res. 1989, 22, 55.

(11) (a) Miller, J. S.; Epstein, A. J. Prog. Inorg. Chem. 1976, 20, 1. (b) Extended Linear Chain Compounds; Miller, J. S., Ed.; Plenum Press: New York, 1982; Vol. 1. (c) Schindler, J. W.; Fukuda, R. C.; Adamson, A. W. J. Am. Chem. Soc. 1982, 104, 3596. (d) Novoa, J. J.; Aullón, G.; Alemany, P.; Alvarez, S. J. Am. Chem. Soc. 1995, 117, 7169. 


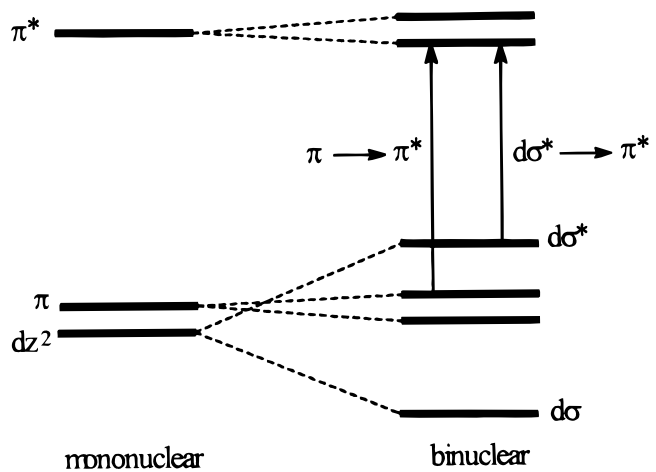

Figure 1. Schematic molecular orbital diagram illustrating $\mathrm{d}^{8}-\mathrm{d}^{8}$ and $\pi-\pi$ interactions in binuclear platinum(II) polypyridine complexes.

$\alpha$-diimine complexes display a variety of low-energy excited states, ${ }^{12-14}$ including $\alpha$-diimine intraligand (IL, $\pi \rightarrow \pi^{*}$ ) transitions of both monomer and dimer and metal-to-ligand charge transfer (MLCT). In addition, when two platinum(II) $\alpha$-diimine units are in close proximity so as to allow metalmetal and ligand-ligand $(\pi-\pi)$ contacts, a low-energy photoluminescence which is red-shifted from the ${ }^{3}$ MLCT emission of mononuclear species is typically observed. The electronic excited-state associated with this emission is denoted as ${ }^{3}\left[\mathrm{~d} \sigma^{*}, \pi^{*}\right]$ (the metal-metal-to-ligand charge transfer (MMLCT) notation is also used in the literature, see Figure $\left.1^{12 a}\right)$. However, it should be noted that the $\mathrm{Pt}(\mathrm{II})-\mathrm{Pt}(\mathrm{II})$ interaction in the ground state is considerably weaker than a normal $\mathrm{Pt}-\mathrm{Pt}$ single bond. Although these $\alpha$-diimine compounds exhibit interesting photophysical properties, they are usually weak emitters or even non-emissive in fluid solution. The tuning of the excited-state properties of the related $\mathrm{Pt}(\mathrm{II})$ diimine dithiolate derivatives have been documented..$^{15}$ In addition, $\mathrm{d}^{8}-\mathrm{d}^{8}$ and $\pi-\pi$ interactions are invoked in oligomerization ${ }^{16-19}$ and excimer formation ${ }^{20-22}$ of mononuclear platinum(II) species in solution, which leads to changes in their photophysical behavior.

Square planar $\mathrm{d}^{8}$ complexes are proposed to be unstable with respect to a $D_{2 d}$ distortion, which is likely to result in nonradiative decay. Many researchers have therefore diverted their attention to the $2,2^{\prime}: 6^{\prime}, 2^{\prime \prime}$-terpyridine (tpy) ligand which shows strong preference for planar geometry. ${ }^{16-19,22,23}$ Several binuclear $\mathrm{d}^{8}-\mathrm{d}^{8}$ complexes of the type $\left[\mathrm{Pt}_{2}(\mathrm{tpy})_{2}(\mu-\mathrm{L})\right]^{n+}(\mathrm{L}=$ bidentate ligand) have been spectroscopically characterized to model

(12) (a) Miskowski, V. M.; Houlding, V. H. Inorg. Chem. 1991, 30, 4446. (b) Houlding, V. H.; Miskowski, V. M. Coord. Chem. Rev. 1991, 111 , 145. (c) Miskowski, V. M.; Houlding, V. H.; Che, C. M.; Wang, Y. Inorg. Chem. 1993, 32, 2518.

(13) Che, C. M.; He, L. Y.; Poon, C. K.; Mak, T. C. W. Inorg. Chem. 1989, 28, 3081.

(14) (a) Zheng, G. Y.; Rillema, D. P.; DePriest, J.; Woods, C. Inorg. Chem. 1998, 37, 3588. (b) Zheng, G. Y.; Rillema, D. P. Inorg. Chem. 1998 37, 1392.

(15) Paw, W.; Cummings, S. D.; Mansour, M. A.; Connick, W. B.; Geiger, D. K.; Eisenberg, R. Coord. Chem. Rev. 1998, 171, 125 .

(16) Arena, G.; Calogero, G.; Campagna, S.; Scolaro, L. M.; Ricevuto, V.; Romeo, R. Inorg. Chem. 1998, 37, 2763.

(17) Hill, M. G.; Bailey, J. A.; Miskowski, V. M.; Gray, H. B. Inorg. Chem. 1996, 35, 4585.

(18) Yip, H. K.; Cheng, L. K.; Cheung K. K.; Che, C. M. J. Chem. Soc., Dalton Trans. 1993, 2933.

(19) Jennette, K. W.; Gill, J. T.; Sadownick, J. A.; Lippard, S. J. J. Am. Chem. Soc. 1976, 98, 6159.

(20) Kunkely, H.; Vogler, A. J. Am. Chem. Soc. 1990, 112, 5625.

(21) Wan, K. T.; Che C. M.; Cho, K. C. J. Chem. Soc., Dalton Trans. $1991,1077$.

(22) Bailey, J. A.; Hill, M. G.; Marsh, R. E.; Miskowski, V. M.; Schaefer, W. P.; Gray, H. B. Inorg. Chem. 1995, 34, 4591. intermolecular interactions in $\mathrm{Pt}(\mathrm{II})$ polypyridine species. ${ }^{24-26}$ We became attracted to $\mathrm{Pt}(\mathrm{II})$ derivatives bearing cyclometalated 6-phenyl-2,2'-bipyridine $\left(\mathrm{L}^{1}\right)$ and related ligands., ${ }^{3,27-30}$ Earlier studies on bidentate analogues employing $\mathrm{C}$-deprotonated 2phenylpyridine and 2-(2'-thienyl)pyridine revealed low-lying metal-to-ligand charge transfer (MLCT) states with interesting photochemical properties. ${ }^{31} \mathrm{We}$ anticipated that the tridentate ligand $\mathrm{L}^{1}$, which favors planar geometry upon cyclometalation, would discourage a $D_{2 d}$ distortion, while the extended $\pi$ system within $\mathrm{L}^{1}$ and the strongly $\sigma$-donating carbanion would increase the energy difference between the ligand field $(d-d)$ and the MLCT states.

Herein is described the preparation and spectroscopic properties of a series of mono- and binuclear cyclometalated platinum(II) complexes derived from 6-phenyl-2,2'-bipyridine. They are photoluminescent at room temperature in fluid solution. The binuclear derivatives are envisaged as models for investigating the photophysics and solid-state structures of cyclometalated $\mathrm{Pt}$ (II) oligomers, and our objective was to characterize the [ $\mathrm{d} \sigma^{*}$, $\left.\pi^{*}\right]$ excited state and $\mathrm{d}^{8}-\mathrm{d}^{8}$ interactions in detail. By systematically varying (1) the length of the bridging bidentate ligand and (2) the electronic nature of substituents on 6-phenyl-2,2'bipyridine, we set out to modify the degree of metal-metal and $\pi-\pi$ interactions and to monitor the effects upon the nature of the excited states.

\section{Experimental Section}

General Procedures. $\mathrm{K}_{2} \mathrm{PtCl}_{4}$ (Strem), bis(diphenylphosphino)methane, -propane, and -pentane ( $\mathrm{dppm}, \mathrm{dppC}_{3}$, and $\mathrm{dppC}_{5}$, respectively, Aldrich), pyrazole (Hpz), and pyridine (py, Aldrich) were used as received. $\mathrm{HL}^{1-6}$ (4-(aryl)-6-phenyl-2,2'-bipyridine; aryl $=\mathrm{H}(\mathbf{1})$, phenyl (2), 4-chlorophenyl (3), 4-tolyl (4), 4-methoxyphenyl (5), 3,4,5trimethoxyphenyl (6)) were prepared by literature methods. ${ }^{32}$ Syntheses of $\left[\mathrm{Pt}\left(\mathrm{L}^{1}\right) \mathrm{Cl}\right](\mathbf{1 a}),\left[\mathrm{Pt}_{2}\left(\mathrm{~L}^{1}\right)_{2}(\mu\right.$-dppm) $]\left(\mathrm{ClO}_{4}\right)_{2}\left(\mathbf{1 b}\left(\mathrm{ClO}_{4}\right)_{2}\right)$, and $\left[\mathrm{Pt}\left(\mathrm{L}^{1}\right)-\right.$ $\mathrm{PPh}_{3} \mathrm{ClO}_{4}\left(\mathbf{8}\left(\mathrm{ClO}_{4}\right)\right)$ have been described previously. ${ }^{29}$ (Caution! Perchlorate salts are potentially explosive and should be handled with care and in small amounts.) Dichloromethane for photophysical studies was washed with concentrated sulfuric acid, $10 \%$ sodium hydrogen carbonate, and water, dried by calcium chloride, and distilled over calcium hydride. Acetonitrile for photophysics was distilled over potassium permanganate and calcium hydride. The other solvents used were of analytical grade.

Physical Measurements and Instrumentation. Fast atom bombardment (FAB) mass spectra were obtained on a Finnigan Mat 95 mass spectrometer. Elemental analyses were performed by Butterworth Laboratory, Teddington, U.K. ${ }^{1} \mathrm{H}$ (in MHz, 300 or 500 ), ${ }^{13} \mathrm{C}(126),{ }^{31} \mathrm{P}$ (202), and ${ }^{195} \mathrm{Pt}$ (107) NMR measurements were performed on a Bruker

(23) Aldridge, T. K.; Stacy, E. M.; McMillin, D. R. Inorg. Chem. 1994, 33,722 .

(24) Bailey, J. A.; Miskowski, V. M.; Gray, H. B. Inorg. Chem. 1993, 32 , 369.

(25) Yip, H. K.; Che, C. M.; Zhou, Z. Y.; Mak, T. C. W. J. Chem. Soc., Chem Commun. 1992, 1369.

(26) Ratilla, E. M. A.; Scott, B. K.; Moxness, M. S.; Kostic, N. M. Inorg. Chem. 1990, 29, 918.

(27) Chan, C. W.; Lai, T. F.; Che, C. M.; Peng, S. M. J. Am. Chem. Soc. 1993, 115, 11245 .

(28) Chan, C. W.; Cheng, L. K.; Che, C. M. Coord. Chem. Rev. 1994, $132,87$.

(29) Cheung, T. C.; Cheung, K. K.; Peng, S. M.; Che, C. M. J. Chem. Soc., Dalton Trans. 1996, 1645.

(30) (a) Tse, M. C.; Cheung, K. K.; Chan, M. C. W.; Che, C. M. Chem. Commun. 1998, 2295. (b) Lai, S. W.; Chan, M. C. W.; Peng, S. M.; Che, C. M. Angew. Chem., Int. Ed. 1999, 38, 669.

(31) (a) Chassot, L.; Müller, E.; von Zelewsky, A. Inorg. Chem. 1984, 23 , 4249. (b) Maestri, M.; Sandrini, D.; Balzani, V.; Chassot, L.; Jolliet, P.; von Zelewsky, A. Chem. Phys. Lett. 1985, 122, 375. (c) Sandrini, D.; Maestri, M.; Balzani, V.; Chassot, L.; von Zelewsky, A. J. Am. Chem. Soc. 1987, 109, 7720.

(32) Kröhnke, F. Synthesis 1976, 1. 
DRX 300 or 500 FT-NMR spectrometer with TMS $\left({ }^{1} \mathrm{H}\right.$ and $\left.{ }^{13} \mathrm{C}\right), \mathrm{H}_{3^{-}}$ $\mathrm{PO}_{4}\left({ }^{31} \mathrm{P}\right)$, and $\mathrm{H}_{2} \mathrm{PtCl}_{6}\left({ }^{195} \mathrm{Pt}\right)$ as references. UV-vis absorption spectra were obtained on a Perkin-Elmer Lambda 19 UV-visible spectrophotometer.

Emission and Lifetime Measurements. Steady-state emission spectra were recorded on a SPEX 1681 Fluorolog-2 series F111AI spectrophotometer. Low-temperature $(77 \mathrm{~K})$ emission spectra for glasses and solid-state samples were recorded in 5-mm diameter quartz tubes, which were placed in a liquid-nitrogen Dewar equipped with quartz windows. The emission spectra were corrected for monochromator and photomultiplier efficiency and for xenon lamp stability.

Sample and standard solutions were degassed with at least three freeze-pump-thaw cycles. The emission quantum yield was measured by the method of Demas and Crosby ${ }^{33}$ with $\left[\mathrm{Ru}(\mathrm{bpy})_{3}\right]\left(\mathrm{PF}_{6}\right)_{2}$ in degassed acetonitrile as the standard $\left(\Phi_{\mathrm{r}}=0.062\right)$.

Emission lifetimes and flash-photolysis measurements were performed with a Quanta Ray DCR-3 pulsed Nd:YAG laser system (pulse output $355 \mathrm{~nm}, 8 \mathrm{~ns}$ ). The emission signals were detected by a Hamamatsu R928 photomultiplier tube and recorded on a Tektronix model 2430 digital oscilloscope. Error limits are estimated: $\lambda( \pm 1 \mathrm{~nm})$; $\tau( \pm 10 \%) ; \phi( \pm 10 \%)$.

Syntheses. $\left[\mathrm{Pt}\left(\mathrm{L}^{2-6}\right) \mathrm{Cl}\right]\left(2 \mathrm{a}-6 \mathrm{a} ; \mathrm{HL}^{2-6}=4\right.$-(aryl)-6-phenyl-2,2'bipyridine; aryl = phenyl (2), 4-chlorophenyl (3), 4-tolyl (4), 4-methoxyphenyl (5), 3,4,5-trimethoxyphenyl (6)). A modification of Constable's method was used. ${ }^{34} \mathrm{~A}$ mixture of $\mathrm{K}_{2} \mathrm{PtCl}_{4}(0.20 \mathrm{~g}, 0.48$ $\mathrm{mmol}$ ) and $\mathrm{L}^{2-6}$ (except $\mathrm{L}^{3}, 0.48 \mathrm{mmol}$ ) in $\mathrm{CH}_{3} \mathrm{CN} / \mathrm{H}_{2} \mathrm{O}(15 / 15 \mathrm{~mL}$ ) was refluxed for $18 \mathrm{~h}$ to give a deep red solution, which was evaporated to dryness. The product was extracted with dichloromethane, and the volume of extract was reduced to $\sim 5 \mathrm{~mL}$. Addition of diethyl ether yielded an orange solid which was recrystallized by vapor diffusion of diethyl ether into an acetonitrile solution to afford a reddish-orange crystalline solid.

2a. Yield: $0.22 \mathrm{~g}, 85 \%$. MS (+ve FAB): $\mathrm{m} / z 538\left(\mathrm{M}^{+}\right)$. Anal. Calcd for $\mathrm{C}_{22} \mathrm{H}_{15} \mathrm{~N}_{2} \mathrm{ClPt}$ : C, 49.12; H, 2.81; N, 5.21. Found: C, 48.98; $\mathrm{H}$, 2.78; N, 5.16. ${ }^{1} \mathrm{H}$ NMR (DMSO- $\left.d_{6}, 300 \mathrm{MHz}\right): \delta 7.06-7.18(\mathrm{~m}, 2 \mathrm{H})$, $7.49-7.63(\mathrm{~m}, 4 \mathrm{H}), 7.82(\mathrm{~d}, 1 \mathrm{H}, J=6.2 \mathrm{~Hz}), 7.92(\mathrm{t}, 1 \mathrm{H}, J=6.4$ $\mathrm{Hz}), 8.11-8.14(\mathrm{~m}, 2 \mathrm{H}), 8.27(\mathrm{~s}, 1 \mathrm{H}), 8.38(\mathrm{t}, 1 \mathrm{H}, J=8.5 \mathrm{~Hz}), 8.53$ $(\mathrm{s}, 1 \mathrm{H}), 8.76(\mathrm{~d}, 1 \mathrm{H}, J=8.1 \mathrm{~Hz}), 8.91(\mathrm{~d}, 1 \mathrm{H}, J=4.4 \mathrm{~Hz}) .{ }^{13} \mathrm{C}\left\{{ }^{1} \mathrm{H}\right\}$ NMR (DMSO- $\left.d_{6}\right)$ : $\delta 116.4,117.0,123.7,124.1,125.1,127.5,128.1$, 129.0, 130.1, 130.2, 134.1, 136.2, 140.3, 142.3, 146.9, 148.0, 150.5, $154.5,156.7,165.3$.

3a. A mixture of $\mathrm{K}_{2} \mathrm{PtCl}_{4}(0.20 \mathrm{~g}, 0.48 \mathrm{mmol})$ and $\mathrm{L}^{3}(0.17 \mathrm{~g}, 0.49$ mmol) in $\mathrm{H}_{2} \mathrm{O} / \mathrm{CH}_{3} \mathrm{CN}(10 / 40 \mathrm{~mL})$ was refluxed for $30 \mathrm{~h}$. An orange suspension was formed, and the reaction mixture was evaporated to dryness. The solid residue was extracted using $N, N$-dimethylformamide (DMF) $(25 \mathrm{~mL} \times 2)$, and the volume of the extract was reduced to $\sim 5$ $\mathrm{mL}$. An orange precipitate appeared upon addition of diethyl ether and was filtered and washed with $\mathrm{H}_{2} \mathrm{O}$ and diethyl ether. Recrystallization by vapor diffusion of diethyl ether into a DMF solution afforded reddish-orange crystals. Yield: $0.19 \mathrm{~g}, 69 \%$. MS (+ve FAB): $\mathrm{m} / z .572$ $\left(\mathrm{M}^{+}\right)$. Anal. Calcd for $\mathrm{C}_{22} \mathrm{H}_{14} \mathrm{~N}_{2} \mathrm{Cl}_{2} \mathrm{Pt}$ : C, 46.17; H, 2.47; N, 4.89. Found: C, 46.10; H, 2.40; N, 4.78. ${ }^{1} \mathrm{H}$ NMR (DMSO- $d_{6}, 300 \mathrm{MHz}$ ): $\delta 7.10-7.16(\mathrm{~m}, 2 \mathrm{H}), 7.51(\mathrm{~d}, 1 \mathrm{H}, J=7.0 \mathrm{~Hz}), 7.69(\mathrm{~d}, 2 \mathrm{H}, J=7.9$ $\mathrm{Hz}), 7.83(\mathrm{~d}, 1 \mathrm{H}, J=7.6 \mathrm{~Hz}), 7.93(\mathrm{t}, 1 \mathrm{H}, J=6.5 \mathrm{~Hz}), 8.18(\mathrm{~d}, 2 \mathrm{H}$, $J=8.0 \mathrm{~Hz}), 8.30(\mathrm{~s}, 1 \mathrm{H}), 8.39(\mathrm{t}, 1 \mathrm{H}, J=7.7 \mathrm{~Hz}), 8.54(\mathrm{~s}, 1 \mathrm{H}), 8.76$ $(\mathrm{d}, 1 \mathrm{H}, J=8.0 \mathrm{~Hz}), 8.92(\mathrm{~d}, 1 \mathrm{H}, J=4.9 \mathrm{~Hz}) .{ }^{13} \mathrm{C}\left\{{ }^{1} \mathrm{H}\right\}$ NMR (DMSO$\left.d_{6}\right)$ : $\delta 115.6,116.4,116.9,123.8,124.2,125.3,128.3,129.1,129.3$, $130.3,134.2,140.4,142.3,146.2,148.3,149.2,149.7,154.5,156.7$, 165.8 .

4a. Yield: $0.23 \mathrm{~g}, 86 \%$. MS (+ve FAB): $\mathrm{m} / z 552\left(\mathrm{M}^{+}\right)$. Anal. Calcd for $\mathrm{C}_{23} \mathrm{H}_{17} \mathrm{~N}_{2} \mathrm{PtCl}$ : C, 50.05; H, 3.10; N, 5.08. Found: C, 49.78; H, 3.02; N, 4.96. ${ }^{1} \mathrm{H}$ NMR (DMSO- $\left.d_{6}, 300 \mathrm{MHz}\right): \delta 2.41$ (s, $\left.3 \mathrm{H}, \mathrm{Me}\right)$, $7.08-7.19(\mathrm{~m}, 2 \mathrm{H}), 7.42(\mathrm{~d}, 2 \mathrm{H}, J=7.9 \mathrm{~Hz}), 7.52(\mathrm{~d}, 1 \mathrm{H}, J=7.2$ $\mathrm{Hz}), 7.84(\mathrm{~d}, 1 \mathrm{H}, J=6.6 \mathrm{~Hz}), 7.94(\mathrm{t}, 1 \mathrm{H}, J=6.4 \mathrm{~Hz}), 8.07$ (d, $2 \mathrm{H}$, $J=8.0 \mathrm{~Hz}), 8.28(\mathrm{~s}, 1 \mathrm{H}), 8.39(\mathrm{t}, 1 \mathrm{H}, J=7.7 \mathrm{~Hz}), 8.53(\mathrm{~s}, 1 \mathrm{H}), 8.78$ $(\mathrm{d}, 1 \mathrm{H}, J=7.9 \mathrm{~Hz}), 8.93(\mathrm{~d}, 1 \mathrm{H}, J=5.2 \mathrm{~Hz}) .{ }^{13} \mathrm{C}\left\{{ }^{1} \mathrm{H}\right\}$ NMR (DMSO-

(33) Demas, J. N.; Crosby, G. A. J. Phys. Chem. 1971, 75, 991.

(34) Constable, E. C.; Henney, R. P. G.; Leese, T. A.; Tocher, D. A. J. Chem. Soc., Chem. Commun. 1990, 513. $\left.d_{6}\right): \delta 20.8(\mathrm{Me}), 115.9,116.5,123.8,124.1,125.1,127.3,128.2,129.7$, $130.2,133.2,134.2,140.3,140.4,142.3,147.0,148.1,150.4,154.5$, $156.8,165.3$

5a. Yield: $0.21 \mathrm{~g}, 77 \%$. MS (+ve FAB): $\mathrm{m} / \mathrm{z} 568\left(\mathrm{M}^{+}\right)$. Anal. Calcd for $\mathrm{C}_{23} \mathrm{H}_{17} \mathrm{~N}_{2} \mathrm{OCIPt}$ : C, 48.64; $\mathrm{H}, 3.02 ; \mathrm{N}, 4.93$. Found: C, 48.85; H, 2.90; N, 4.87. ${ }^{1} \mathrm{H}$ NMR (DMSO- $\left.d_{6}, 300 \mathrm{MHz}\right): \delta 3.88$ (s, $3 \mathrm{H}, \mathrm{OMe}$ ), $7.15(\mathrm{~d}, 2 \mathrm{H}, J=6.6 \mathrm{~Hz}), 7.50-7.62(\mathrm{~m}, 2 \mathrm{H}), 7.83(\mathrm{~d}, 1 \mathrm{H}, J=7.6$ $\mathrm{Hz}), 7.92(\mathrm{t}, 1 \mathrm{H}, J=6.4 \mathrm{~Hz}), 8.01(\mathrm{~d}, 1 \mathrm{H}, J=8.7 \mathrm{~Hz}), 8.14(\mathrm{~d}, 2 \mathrm{H}$, $J=8.7 \mathrm{~Hz}), 8.25(\mathrm{~s}, 1 \mathrm{H}), 8.35-8.40(\mathrm{~m}, 1 \mathrm{H}), 8.50(\mathrm{~s}, 1 \mathrm{H}), 8.76(\mathrm{~d}$, $1 \mathrm{H}, J=8.0 \mathrm{~Hz}$ ), $8.92(\mathrm{~d}, 1 \mathrm{H}, J=5.0 \mathrm{~Hz}) .{ }^{13} \mathrm{C}\left\{{ }^{1} \mathrm{H}\right\}$ NMR (DMSO$\left.d_{6}\right): \delta 56.2(\mathrm{OMe}), 115.4,115.5,118.9,124.6,128.0,129.1,129.4$, 129.6, 130.0, 130.1, 141.1, 143.2, 148.0, 150.2, 150.9, 155.2, 157.7, 161.4, 162.1, 166.0.

6a. Yield: $0.21 \mathrm{~g}, 69 \%$. MS (+ve FAB): $m / z 628\left(\mathrm{M}^{+}\right)$. Anal. Calcd for $\mathrm{C}_{25} \mathrm{H}_{21} \mathrm{~N}_{2} \mathrm{O}_{3} \mathrm{ClPt}$ : C, 47.81; H, 3.37; N, 4.46. Found: C, 47.90; H, 3.25 ; N, 4.29. ${ }^{1} \mathrm{H}$ NMR (DMSO- $d_{6}, 300 \mathrm{MHz}$ ): $\delta 3.76$ (s, $3 \mathrm{H}, p$-OMe), $3.98(\mathrm{~s}, 6 \mathrm{H}, m-\mathrm{OMe}), 7.11-7.17(\mathrm{~m}, 2 \mathrm{H}), 7.34(\mathrm{~s}, 2 \mathrm{H}), 7.51(\mathrm{~d}, 1 \mathrm{H}, J$ $=6.7 \mathrm{~Hz}), 7.85(\mathrm{~d}, 1 \mathrm{H}, J=7.2 \mathrm{~Hz}), 7.92-7.96(\mathrm{~m}, 1 \mathrm{H}), 8.23(\mathrm{~s}, 1 \mathrm{H})$, $8.40-8.44(\mathrm{~m}, 2 \mathrm{H}), 8.74(\mathrm{~d}, 1 \mathrm{H}, J=7.6 \mathrm{~Hz}), 8.92-8.94(\mathrm{~m}, 1 \mathrm{H})$. ${ }^{13} \mathrm{C}\left\{{ }^{1} \mathrm{H}\right\}$ NMR (DMSO- $\left.d_{6}\right): \delta 55.4(m-\mathrm{OMe}), 59.1$ ( $p$-OMe), 115.5, 116.0, 116.9, 122.8, 123.2, 124.3, 127.3, 129.3, 130.8, 132.6, 133.2, $138.8,139.4,145.9,147.1,149.8,152.4,153.3,155.8,164.2$.

$\left[\mathrm{Pt}\left(\mathrm{L}^{1}\right)\right.$ py] $\mathrm{ClO}_{4}, 7\left(\mathrm{ClO}_{4}\right)$. A mixture of $\left[\mathrm{Pt}\left(\mathrm{L}^{1}\right) \mathrm{Cl}\right](0.25 \mathrm{~g}, 0.54$ $\mathrm{mmol})$ and excess pyridine $(0.20 \mathrm{~g}, 2.70 \mathrm{mmol})$ in $\mathrm{CH}_{3} \mathrm{CN} / \mathrm{CH}_{3} \mathrm{OH}$ $(20 / 20 \mathrm{~mL})$ was stirred for $3 \mathrm{~h}$ at room temperature. Excess $\mathrm{LiClO}_{4}$ $(0.2 \mathrm{~g})$ was added to the resultant mixture, which was stirred for $5 \mathrm{~h}$ and then filtered and evaporated to $\sim 5 \mathrm{~mL}$. Addition of diethyl ether yielded a yellow-brown solid which was filtered and washed with diethyl ether. Recrystallization by vapor diffusion of diethyl ether into an acetonitrile solution yielded $0.28 \mathrm{~g}(86 \%)$ of yellow crystals. MS (+ve FAB): $m / z 505\left(\mathrm{M}^{+}\right)$. Anal. Calcd for $\mathrm{C}_{21} \mathrm{H}_{16} \mathrm{~N}_{3} \mathrm{O}_{4} \mathrm{ClPt}$ : C, 41.70; $\mathrm{H}, 2.67 ; \mathrm{N}, 6.95$. Found: $\mathrm{C}, 41.95 ; \mathrm{H}, 2.55 ; \mathrm{N}, 6.87 .{ }^{1} \mathrm{H}$ NMR (DMSO$\left.d_{6}, 300 \mathrm{MHz}\right): \delta 6.28(\mathrm{~d}, 1 \mathrm{H}, J=7.5 \mathrm{~Hz}), 7.03-7.15(\mathrm{~m}, 2 \mathrm{H}), 7.70-$ $7.85(\mathrm{~m}, 4 \mathrm{H}), 8.07(\mathrm{~d}, 2 \mathrm{H}, J=6.9 \mathrm{~Hz}), 8.15-8.43(\mathrm{~m}, 4 \mathrm{H}), 8.58(\mathrm{~d}$, $1 \mathrm{H}, J=7.8 \mathrm{~Hz}$ ), $9.09(\mathrm{~d}, 2 \mathrm{H}, J=5.1 \mathrm{~Hz}) .{ }^{13} \mathrm{C}\left\{{ }^{1} \mathrm{H}\right\}$ NMR (DMSO$\left.d_{6}\right): \delta 120.3,124.2,125.0,125.6,126.2,128.1,129.2,131.4,132.4$, $140.2,141.4,141.7,142.3,147.5,149.4,153.4,155.0,156.6,165.5$.

$\left[\mathrm{Pt}_{2}\left(\mathrm{~L}^{2-6}\right)_{2}(\mu\right.$-dppm $\left.)\right]\left(\mathrm{ClO}_{4}\right)_{2}, \mathbf{2 b}-\mathbf{6 b}\left(\mathrm{ClO}_{4}\right)_{2}$. A mixture of [Pt$\left(\mathrm{L}^{2-6}\right) \mathrm{Cl}$ ] (except $\left.\mathrm{L}^{3}, 0.32 \mathrm{mmol}\right)$ and dppm $(0.06 \mathrm{~g}, 0.16 \mathrm{mmol})$ in $\mathrm{CH}_{3} \mathrm{CN} / \mathrm{CH}_{3} \mathrm{OH}(15 / 15 \mathrm{~mL})$ was stirred for $12 \mathrm{~h}$ under a nitrogen atmosphere. The resultant solution was filtered and evaporated to $\sim 5$ $\mathrm{mL}$. Addition of excess aqueous $\mathrm{LiClO}_{4}$ afforded a bright orange solid, which was filtered and washed with water and diethyl ether. Recrystallization by vapor diffusion of diethyl ether into an acetonitrile solution yielded an orangish-red crystalline solid.

2b(ClO $\left.)_{4}\right)_{2}$. Yield: $0.20 \mathrm{~g}, 79 \%$. MS (+ve FAB): $\mathrm{m} / \mathrm{z} 1489\left(\mathrm{M}^{+}+\right.$ $\left.\mathrm{ClO}_{4}\right), 1389\left(\mathrm{M}^{+}\right)$. Anal. Calcd for $\mathrm{C}_{69} \mathrm{H}_{52} \mathrm{~N}_{4} \mathrm{O}_{8} \mathrm{Pt}_{2} \mathrm{Cl}_{2} \mathrm{P}_{2}: \mathrm{C}, 52.18 ; \mathrm{H}$, 3.30; N, 3.53. Found: C, 52.08; H, 3.27; N, 3.48. ${ }^{1} \mathrm{H}$ NMR (DMSO$\left.d_{6}, 300 \mathrm{MHz}\right): \delta 5.26\left(\right.$ broad t $\left., 2 \mathrm{H},{ }^{2} J(\mathrm{PH})=12 \mathrm{~Hz}, \mathrm{PCH}_{2} \mathrm{P}\right), 6.16(\mathrm{~s}$, $2 \mathrm{H}), 6.40-6.45(\mathrm{~m}, 2 \mathrm{H}), 6.59-6.69(\mathrm{~m}, 6 \mathrm{H}), 7.36-7.60(\mathrm{~m}, 20 \mathrm{H})$, 7.76-7.79 (m, 8H), 7.97-8.04 (m, 4H), $8.23(\mathrm{~s}, 2 \mathrm{H}), 8.41-8.52(\mathrm{~m}$, $6 \mathrm{H}) .{ }^{13} \mathrm{C}\left\{{ }^{1} \mathrm{H}\right\}$ NMR (DMSO- $\left.d_{6}\right): \delta 19.4\left(\mathrm{t},{ }^{1} J(\mathrm{PC})=30.1 \mathrm{~Hz}, \mathrm{PCH}_{2} \mathrm{P}\right)$, 116.7, 117.0, 124.4-134.9, 137.5, 140.0, 146.7, 150.7, 153.2, 153.4, 156.4, 162.3. ${ }^{31} \mathrm{P}\left\{{ }^{1} \mathrm{H}\right\} \mathrm{NMR}\left(\mathrm{CD}_{3} \mathrm{CN}\right): \delta 19.36\left({ }^{1} J(\mathrm{PtP})=4114 \mathrm{~Hz}\right.$, $\left.{ }^{3} J(\mathrm{PtP})=87 \mathrm{~Hz}\right) \cdot{ }^{195} \mathrm{Pt} \mathrm{NMR}\left(\mathrm{CD}_{3} \mathrm{CN}\right): \delta-4095\left(\mathrm{~d},{ }^{1} J(\mathrm{PPt})=4111\right.$ $\mathrm{Hz})$.

3b(ClO $)_{2}$. Dppm $(0.08 \mathrm{~g}, 0.20 \mathrm{mmol})$ in $\mathrm{CH}_{3} \mathrm{CN}(10 \mathrm{~mL})$ was added dropwise to a solution of $\left[\mathrm{Pt}\left(\mathrm{L}^{3}\right) \mathrm{Cl}\right](0.23 \mathrm{~g}, 0.40 \mathrm{mmol})$ in $\mathrm{CH}_{3} \mathrm{CN} /$ $\mathrm{CH}_{2} \mathrm{Cl}_{2}(10 / 10 \mathrm{~mL})$ and stirred for $24 \mathrm{~h}$ under a nitrogen atmosphere to afford a clear red-orange solution. After addition of methanolic $\mathrm{LiClO}_{4}(0.2 \mathrm{~g})$, the solution was further stirred for $2 \mathrm{~h}$ and then reduced to $\sim 5 \mathrm{~mL}$. Addition of diethyl ether gave a red-orange solid which was filtered and washed with water and diethyl ether. Recrystallization by vapor diffusion of diethyl ether into an acetonitrile solution yielded an orangish-red crystalline solid. Yield: $0.23 \mathrm{~g}, 69 \%$. MS (+ve FAB): $m / z, 1558\left(\mathrm{M}^{+}+\mathrm{ClO}_{4}\right), 1458\left(\mathrm{M}^{+}\right)$. Anal. Calcd for $\mathrm{C}_{69} \mathrm{H}_{50} \mathrm{~N}_{4} \mathrm{O}_{8-}$ $\mathrm{Pt}_{2} \mathrm{Cl}_{4} \mathrm{P}_{2}$ : C, 50.01; H, 3.04; N, 3.38. Found: C, 49.95; H, 2.95; N, 3.32. ${ }^{1} \mathrm{H}$ NMR (DMSO- $\left.d_{6}, 300 \mathrm{MHz}\right): \delta 5.22\left(\right.$ broad t $, 2 \mathrm{H},{ }^{2} J(\mathrm{PH})=$ $\left.12 \mathrm{~Hz}, \mathrm{PCH}_{2} \mathrm{P}\right), 6.23-6.39(\mathrm{~m}, 4 \mathrm{H}), 6.54-6.73(\mathrm{~m}, 6 \mathrm{H}), 7.40-7.61$ (m, 18H), 7.78-7.87 (m, 8H), 7.99-8.04 (m, 4H), 8.28-8.37 (m, 6H), $8.51(\mathrm{~d}, 2 \mathrm{H}, J=8.1 \mathrm{~Hz}) .{ }^{13} \mathrm{C}\left\{{ }^{1} \mathrm{H}\right\}$ NMR (DMSO- $\left.d_{6}\right): \delta 20.3\left(\mathrm{t},{ }^{1} J(\mathrm{PC})\right.$ 
$\left.=32.1 \mathrm{~Hz}, \mathrm{PCH}_{2} \mathrm{P}\right), 116.3,116.9,124.4-139.9,146.6,150.9,151.7$, 153.3, 156.5, 162.4. ${ }^{31} \mathrm{P}\left\{{ }^{1} \mathrm{H}\right\} \mathrm{NMR}\left(\mathrm{CD}_{3} \mathrm{CN}\right): \delta 19.31\left({ }^{1} J(\mathrm{PtP})=4124\right.$ $\left.\mathrm{Hz},{ }^{3} J(\mathrm{PtP})=81 \mathrm{~Hz}\right) .{ }^{195} \mathrm{Pt} \mathrm{NMR}\left(\mathrm{CD}_{3} \mathrm{CN}\right): \delta-4087\left(\mathrm{~d},{ }^{1} J(\mathrm{PPt})=\right.$ $4129 \mathrm{~Hz})$.

4b(ClO $\left.)_{4}\right)_{2}$. Yield: $0.20 \mathrm{~g}, 77 \%$. MS (+ve FAB): $\mathrm{m} / z 1517\left(\mathrm{M}^{+}+\right.$ $\left.\mathrm{ClO}_{4}\right), 1417\left(\mathrm{M}^{+}\right)$. Anal. Calcd for $\mathrm{C}_{71} \mathrm{H}_{56} \mathrm{~N}_{4} \mathrm{O}_{8} \mathrm{Pt}_{2} \mathrm{Cl}_{2} \mathrm{P}_{2}: \mathrm{C}, 52.76 ; \mathrm{H}$, 3.49; N, 3.47. Found: C, 52.70; $\mathrm{H}, 3.42 ; \mathrm{N}, 3.45 .{ }^{1} \mathrm{H}$ NMR $\left(\mathrm{CD}_{3} \mathrm{CN}\right.$, $500 \mathrm{MHz}): \delta 2.42(\mathrm{~s}, 6 \mathrm{H}, \mathrm{Me}), 4.85\left(\right.$ broad t, $2 \mathrm{H},{ }^{2} J(\mathrm{PH})=13 \mathrm{~Hz}$, $\left.\mathrm{PCH}_{2} \mathrm{P}\right), 6.22(\mathrm{~s}, 2 \mathrm{H}), 6.41(\mathrm{t}, 2 \mathrm{H}, J=6.9 \mathrm{~Hz}), 6.56(\mathrm{t}, 2 \mathrm{H}, J=6.1$ $\mathrm{Hz}), 6.62(\mathrm{t}, 2 \mathrm{H}, J=7.3 \mathrm{~Hz}), 6.72-6.73(\mathrm{~m}, 2 \mathrm{H}), 7.08(\mathrm{~d}, 2 \mathrm{H}, J=$ $7.1 \mathrm{~Hz}), 7.18(\mathrm{~d}, 4 \mathrm{H}, J=7.8 \mathrm{~Hz}), 7.41-7.57(\mathrm{~m}, 16 \mathrm{H}), 7.59(\mathrm{~s}, 2 \mathrm{H})$, $7.70-7.87(\mathrm{~m}, 8 \mathrm{H}), 8.02(\mathrm{~d}, 2 \mathrm{H}, J=7.8 \mathrm{~Hz}), 8.25-8.33$ (broad s, $4 \mathrm{H}) .{ }^{13} \mathrm{C}\left\{{ }^{1} \mathrm{H}\right\}$ NMR $\left(\mathrm{CD}_{3} \mathrm{CN}\right): \delta 21.3\left(\mathrm{t},{ }^{1} J(\mathrm{PC})=31.4 \mathrm{~Hz}, \mathrm{PCH}_{2} \mathrm{P}\right)$, $21.5(\mathrm{Me}), 117.5,117.7,125.0-133.3,139.1,141.0,142.9,147.9$, 152.4, 154.5, 155.0, 157.8, 163.8. ${ }^{31} \mathrm{P}\left\{{ }^{1} \mathrm{H}\right\}$ NMR $\left(\mathrm{CD}_{3} \mathrm{CN}\right): \delta 19.40$ $\left({ }^{1} J(\mathrm{PtP})=4111 \mathrm{~Hz},{ }^{3} J(\mathrm{PtP})=83 \mathrm{~Hz}\right) .{ }^{195} \mathrm{Pt} \mathrm{NMR}\left(\mathrm{CD}_{3} \mathrm{CN}\right): \delta-4091$ $\left(\mathrm{d},{ }^{1} J(\mathrm{PPt})=4113 \mathrm{~Hz}\right)$.

5b $\left(\mathbf{C l O}_{4}\right)_{2}$. Yield: $0.19 \mathrm{~g}, 72 \%$. MS (+ve FAB): $\mathrm{m} / \mathrm{z} 1549\left(\mathrm{M}^{+}+\right.$ $\left.\mathrm{ClO}_{4}\right), 1449\left(\mathrm{M}^{+}\right)$. Anal. Calcd for $\mathrm{C}_{71} \mathrm{H}_{56} \mathrm{~N}_{4} \mathrm{O}_{10} \mathrm{Pt}_{2} \mathrm{Cl}_{2} \mathrm{P}_{2}$ : C, 51.74; $\mathrm{H}$, 3.42; N, 3.40. Found: C, 51.80; H, 3.46; N, 3.48. ${ }^{1} \mathrm{H}$ NMR $\left(\mathrm{CD}_{3} \mathrm{CN}\right.$, $300 \mathrm{MHz}): \delta 3.88(\mathrm{~s}, 6 \mathrm{H}, \mathrm{OMe}), 4.84\left(\operatorname{broad} \mathrm{t}, 2 \mathrm{H},{ }^{2} J(\mathrm{PH})=13 \mathrm{~Hz}\right.$, $\left.\mathrm{PCH}_{2} \mathrm{P}\right), 6.15(\mathrm{~s}, 2 \mathrm{H}), 6.20-6.41(\mathrm{~m}, 2 \mathrm{H}), 6.55-6.60(\mathrm{~m}, 4 \mathrm{H}), 6.71-$ $6.80(\mathrm{~m}, 2 \mathrm{H}), 6.88(\mathrm{~d}, 4 \mathrm{H}, J=8.9 \mathrm{~Hz}), 7.07-7.14(\mathrm{~m}, 2 \mathrm{H}), 7.42-$ $7.59(\mathrm{~m}, 18 \mathrm{H}), 7.70-8.04(\mathrm{~m}, 10 \mathrm{H}), 8.25-8.47$ (broad s, $4 \mathrm{H}) .{ }^{13} \mathrm{C}\left\{{ }^{1} \mathrm{H}\right\}$ NMR $\left(\mathrm{CD}_{3} \mathrm{CN}\right): \delta 21.6\left(\mathrm{t},{ }^{1} J(\mathrm{PC})=31.3 \mathrm{~Hz}, \mathrm{PCH}_{2} \mathrm{P}\right), 56.3(\mathrm{OMe})$, 115.6, 116.5, 117.0, 125.0-135.4, 139.0, 140.9, 147.9, 152.3, 154.2, 154.4, 157.8, 163.2, 163.6. ${ }^{31} \mathrm{P}\left\{{ }^{1} \mathrm{H}\right\}$ NMR $\left(\mathrm{CD}_{3} \mathrm{CN}\right): \delta 19.52\left({ }^{1} J(\mathrm{PtP})\right.$ $\left.=4108 \mathrm{~Hz},{ }^{3} J(\mathrm{PtP})=85 \mathrm{~Hz}\right) \cdot{ }^{195} \mathrm{Pt} \mathrm{NMR}\left(\mathrm{CD}_{3} \mathrm{CN}\right): \delta-4087(\mathrm{~d}$, $\left.{ }^{1} J(\mathrm{PPt})=4110 \mathrm{~Hz}\right)$.

6b $\left(\mathrm{ClO}_{4}\right)_{2}$. Yield: $0.21 \mathrm{~g}, 74 \%$. MS (+ve FAB): $\mathrm{m} / \mathrm{z} 1669\left(\mathrm{M}^{+}+\right.$ $\left.\mathrm{ClO}_{4}\right), 1569\left(\mathrm{M}^{+}\right)$. Anal. Calcd for $\mathrm{C}_{75} \mathrm{H}_{64} \mathrm{~N}_{4} \mathrm{O}_{14} \mathrm{Pt}_{2} \mathrm{Cl}_{2} \mathrm{P}_{2}: \mathrm{C}, 50.94 ; \mathrm{H}$, 3.65; N, 3.17. Found: C, 50.80; $\mathrm{H}, 3.52 ; \mathrm{N}, 3.10 .{ }^{1} \mathrm{H}$ NMR $\left(\mathrm{CD}_{3} \mathrm{CN}\right.$, $300 \mathrm{MHz}): \delta 3.79(\mathrm{~s}, 6 \mathrm{H}, p-\mathrm{OMe}), 3.82(\mathrm{~s}, 12 \mathrm{H}, m-\mathrm{OMe}), 4.85(\mathrm{t}$, $\left.2 \mathrm{H},{ }^{2} J(\mathrm{PH})=13.6 \mathrm{~Hz}, \mathrm{PCH}_{2} \mathrm{P}\right), 6.25(\mathrm{~s}, 2 \mathrm{H}), 6.36(\mathrm{t}, 2 \mathrm{H}, J=7.0 \mathrm{~Hz})$, $6.50(\mathrm{t}, 2 \mathrm{H}, J=7.5 \mathrm{~Hz}), 6.69(\mathrm{t}, 2 \mathrm{H}, J=6.3 \mathrm{~Hz}), 6.79(\mathrm{~s}, 6 \mathrm{H}), 7.32$ $(\mathrm{d}, 2 \mathrm{H}, J=7.6 \mathrm{~Hz}), 7.44-7.94(\mathrm{~m}, 22 \mathrm{H}), 8.15(\mathrm{~d}, 2 \mathrm{H}, J=8.1 \mathrm{~Hz})$, 8.28 (broad s, 4H). ${ }^{13} \mathrm{C}\left\{{ }^{1} \mathrm{H}\right\}$ NMR (DMSO- $\left.d_{6}\right): \delta 19.9\left(\mathrm{t},{ }^{1} J(\mathrm{PC})=\right.$ $\left.30.5 \mathrm{~Hz}, \mathrm{PCH}_{2} \mathrm{P}\right), 56.1$ ( $m$-OMe), 59.9 (p-OMe), 105.3, 116.3, 116.9, 124.3-140.1, 146.7, 150.9, 153.0, 153.1, 153.3, 156.7, 162.2. ${ }^{31} \mathrm{P}\left\{{ }^{1} \mathrm{H}\right\}$ $\mathrm{NMR}\left(\mathrm{CD}_{3} \mathrm{CN}\right): \delta 19.65\left({ }^{1} J(\mathrm{PtP})=4134 \mathrm{~Hz},{ }^{3} J(\mathrm{PtP})=78 \mathrm{~Hz}\right) \cdot{ }^{195} \mathrm{Pt}$ $\operatorname{NMR}\left(\mathrm{CD}_{3} \mathrm{CN}\right): \delta-4083\left(\mathrm{~d},{ }^{1} J(\mathrm{PPt})=4125 \mathrm{~Hz}\right)$.

$\left[\mathrm{Pt}_{2}\left(\mathbf{L}^{1}\right)_{2}(\mu-\mathrm{pz})\right] \mathbf{X}, \mathbf{9}(\mathbf{X})\left(\mathbf{X}=\mathbf{C l O}_{4}\right.$ and $\left.\mathbf{P F}_{6}\right)$. A mixture of [Pt$\left.\left(\mathrm{L}^{1}\right) \mathrm{Cl}\right](0.23 \mathrm{~g}, 0.50 \mathrm{mmol})$, pyrazole $(\mathrm{Hpz})(0.02 \mathrm{~g}, 0.25 \mathrm{mmol})$, and potassium tert-butoxide $(0.03 \mathrm{~g}, 0.25 \mathrm{mmol})$ in $\mathrm{CH}_{3} \mathrm{CN} / \mathrm{CH}_{3} \mathrm{OH}(20 /$ $10 \mathrm{~mL}$ ) was heated at $60^{\circ} \mathrm{C}$ under a nitrogen atmosphere for $10 \mathrm{~h}$. The orange suspension gradually became a clear red-orange solution and was allowed to cool to room temperature. A methanolic solution of $\mathrm{LiClO}_{4}$ or $\mathrm{NH}_{4} \mathrm{PF}_{6}(1.5 \mathrm{mmol}$ in $10 \mathrm{~mL})$ was added, and the resultant mixture was stirred for $1 \mathrm{~h}$, after which the solvent was evaporated to $\sim 5 \mathrm{~mL}$. Addition of diethyl ether yielded an orange solid which was recrystallized by vapor diffusion of diethyl ether into an acetonitrile solution. Yield: $0.14 \mathrm{~g}, 55 \%$ for $\mathbf{9}\left(\mathrm{ClO}_{4}\right) ; 0.14 \mathrm{~g}, 53 \%$ for $\mathbf{9}\left(\mathrm{PF}_{6}\right)$. Anal. Calcd for $9\left(\mathrm{ClO}_{4}\right), \mathrm{C}_{35} \mathrm{H}_{25} \mathrm{~N}_{6} \mathrm{O}_{4} \mathrm{Pt}_{2} \mathrm{Cl}: \mathrm{C}, 41.24 ; \mathrm{H}, 2.47 ; \mathrm{N}, 8.25$. Found: C, 41.18; H, 2.63; N, 8.42. Calcd for $9\left(\mathrm{PF}_{6}\right), \mathrm{C}_{35} \mathrm{H}_{25} \mathrm{~N}_{6} \mathrm{Pt}_{2} \mathrm{PF}_{6}$ : C, 39.48; H, 2.37; N, 7.89. Found: C, 39.42; H, 2.52; N, 8.05.

Data for $9\left(\mathrm{ClO}_{4}\right)$ and $9\left(\mathbf{P F}_{6}\right)$. MS (+ve FAB): $\mathrm{m} / z, 919\left(\mathrm{M}^{+}\right)$. ${ }^{1} \mathrm{H}$ NMR (DMSO- $\left.d_{6}, 300 \mathrm{MHz}\right): \delta 6.78-6.80(\mathrm{~m}, 4 \mathrm{H}), 6.88-7.12(\mathrm{~m}$, $5 \mathrm{H}), 7.48(\mathrm{~d}, 2 \mathrm{H}, J=7.2 \mathrm{~Hz}), 7.83(\mathrm{~d}, 2 \mathrm{H}, J=7.7 \mathrm{~Hz}), 7.93-8.09$ $(\mathrm{m}, 8 \mathrm{H}), 8.25-8.36(\mathrm{~m}, 4 \mathrm{H}),{ }^{13} \mathrm{C}\left\{{ }^{1} \mathrm{H}\right\}$ NMR (DMSO- $\left.d_{6}\right): \delta 107.4$, $119.2,119.3,123.4,124.3,125.0,127.1,130.5,133.7,140.4,140.8$, $141.1,141.7,146.4,151.0,154.3,155.4,165.1$.

$\left[\mathrm{Pt}_{2}\left(\mathrm{~L}^{1}\right)_{2}\left(\mu\right.\right.$-dppC $\left.\left.\mathrm{C}_{3}\right)\right]\left(\mathrm{ClO}_{4}\right)_{2}, \mathbf{1 0}\left(\mathrm{ClO}_{4}\right)_{2}$. The procedure for $\mathbf{1 b}\left(\mathrm{ClO}_{4}\right)_{2}$ was adopted using $\mathrm{Pt}\left(\mathrm{L}^{1}\right) \mathrm{Cl}(0.18 \mathrm{~g}, 0.38 \mathrm{mmol})$ and bis(diphenylphosphino)propane $(0.08 \mathrm{~g}, 0.19 \mathrm{mmol})$ to yield $0.18 \mathrm{~g}(63 \%)$ of a yellow crystalline solid. MS (+ve FAB): $m / z, 1365\left(\mathrm{M}^{+}+\mathrm{ClO}_{4}\right), 1265\left(\mathrm{M}^{+}\right)$. Anal. Calcd for $\mathrm{C}_{59} \mathrm{H}_{48} \mathrm{~N}_{4} \mathrm{O}_{8} \mathrm{Pt}_{2} \mathrm{Cl}_{2} \mathrm{P}_{2}$ : C, 48.40; H, 3.30; N, 3.83. Found: $\mathrm{C}, 48.25 ; \mathrm{H}, 3.26 ; \mathrm{N}, 3.76 .{ }^{1} \mathrm{H}$ NMR $\left(\mathrm{CD}_{3} \mathrm{CN}, 500 \mathrm{MHz}\right): \delta$ $3.09-3.13\left(\mathrm{~m}, 2 \mathrm{H}, \mathrm{PCH}_{2} \mathrm{CH}_{2}\right), 3.33-3.41\left(\mathrm{~m}, 4 \mathrm{H}, \mathrm{PCH}_{2}\right), 6.25-6.33$ $(\mathrm{m}, 4 \mathrm{H}), 6.61(\mathrm{t}, 2 \mathrm{H}, J=7.4 \mathrm{~Hz}), 6.74-6.75(\mathrm{~m}, 2 \mathrm{H}), 6.86(\mathrm{t}, 2 \mathrm{H}, J$ $=6.5 \mathrm{~Hz}), 7.04(\mathrm{~d}, 2 \mathrm{H}, J=7.7 \mathrm{~Hz}), 7.35-7.38(\mathrm{~m}, 8 \mathrm{H}), 7.43-7.47$ $(\mathrm{m}, 4 \mathrm{H}), 7.58(\mathrm{~d}, 2 \mathrm{H}, J=8.1 \mathrm{~Hz}), 7.72-7.76(\mathrm{~m}, 8 \mathrm{H}), 7.84-7.97(\mathrm{~m}$,
Table 1. Crystal Data

\begin{tabular}{lll}
\hline & \multicolumn{1}{c}{$\mathbf{4 b}\left(\mathrm{ClO}_{4}\right)_{2} \cdot 5 \mathrm{H}_{2} \mathrm{O}$} & \multicolumn{1}{c}{$\mathbf{9}\left(\mathrm{PF}_{6}\right)$} \\
\hline formula & $\mathrm{C}_{71} \mathrm{H}_{66} \mathrm{~N}_{4} \mathrm{Cl}_{2} \mathrm{O}_{13} \mathrm{P}_{2} \mathrm{Pt}_{2}$ & $\mathrm{C}_{35} \mathrm{H}_{25} \mathrm{~N}_{6} \mathrm{PF}_{6} \mathrm{Pt}_{2}$ \\
fw & 1706.36 & 1064.77 \\
cryst system & monoclinic & monoclinic \\
space group & $C 2 / \mathrm{c}$ & $C 2 / \mathrm{c}$ \\
color & orange & orange \\
cryst size, mm & $0.35 \times 0.35 \times 0.35$ & $0.20 \times 0.30 \times 0.40$ \\
$a, \AA$ & $31.338(8)$ & $15.339(2)$ \\
$b, \AA$ & $21.767(5)$ & $13.309(1)$ \\
$c, \AA$ & $27.515(6)$ & $16.264(5)$ \\
$\beta$, deg & $123.37(2)$ & $103.03(2)$ \\
$V, \AA^{3}$ & $15675(6)$ & $3235(1)$ \\
$Z$ & 8 & 4 \\
$D_{\mathrm{c}}, \mathrm{g} \mathrm{cm}{ }^{-3}$ & 1.447 & 2.186 \\
$\mu, \mathrm{cm}{ }^{-1}$ & 37.68 & 88.50 \\
$F(000)$ & 6752 & 2008 \\
$R,{ }^{a} R_{\mathrm{w}}{ }^{b}$ & $0.059,0.058$ & $0.025,0.027$ \\
$\mathrm{GoF}^{c}$ & 1.75 & 1.48 \\
residual $\rho, \mathrm{e} \AA^{-3}$ & $-0.57,+0.87$ & $-0.75,+1.50$ \\
${ }^{a} R=\Sigma|| F_{\mathrm{o}}|-| F_{\mathrm{c}}|| \Sigma\left|F_{\mathrm{o}}\right| .{ }^{b} R_{\mathrm{w}}=\left[\Sigma w\left(\left|F_{\mathrm{o}}\right|\right.\right.$ & $\left.\left.-\left|F_{\mathrm{c}}\right|\right)^{2} / \Sigma w\left|F_{\mathrm{o}}\right|^{2}\right]^{1 / 2} \cdot{ }^{c} \mathrm{GoF}$ \\
$=$ &
\end{tabular}

$6 \mathrm{H}), 8.08(\mathrm{t}, 2 \mathrm{H}, J=8.0 \mathrm{~Hz}) .{ }^{13} \mathrm{C}\left\{{ }^{1} \mathrm{H}\right\} \operatorname{NMR}\left(\mathrm{CD}_{3} \mathrm{CN}\right): \delta 26.1(\mathrm{dd}$, $\left.{ }^{1} J(\mathrm{PC})=37.2 \mathrm{~Hz},{ }^{3} J(\mathrm{PC})=15.6 \mathrm{~Hz}, \mathrm{PCH}_{2}\right), 26.9\left(\mathrm{t},{ }^{2} J(\mathrm{PC})=7.2\right.$ $\left.\mathrm{Hz}, \mathrm{PCH}_{2} \mathrm{CH}_{2}\right), 120.9,121.2,125.4-138.6,141.9,143.7,148.3,152.2$, $154.4,158.9,164.1 .{ }^{31} \mathrm{P}\left\{{ }^{1} \mathrm{H}\right\} \mathrm{NMR}\left(\mathrm{CD}_{3} \mathrm{CN}\right): \delta 19.71\left({ }^{1} J(\mathrm{PtP})=3990\right.$ $\mathrm{Hz})$.

$\left[\mathrm{Pt}_{2}\left(\mathrm{~L}^{1}\right)_{2}\left(\mu\right.\right.$-dppC $\left.\left.\mathrm{C}_{5}\right)\right]\left(\mathrm{ClO}_{4}\right)_{2}, \mathbf{1 1}\left(\mathrm{ClO}_{4}\right)_{2}$. The procedure for $\mathbf{1 b}\left(\mathrm{ClO}_{4}\right)_{2}$ was adopted using $\mathrm{Pt}\left(\mathrm{L}^{1}\right) \mathrm{Cl}(0.18 \mathrm{~g}, 0.40 \mathrm{mmol})$ with bis(diphenylphosphino)pentane $(0.09 \mathrm{~g}, 0.20 \mathrm{mmol})$ to afford $0.19 \mathrm{~g}(64 \%)$ of a yellow crystalline solid. MS (+ve FAB): m/z. $1393\left(\mathrm{M}^{+}+\mathrm{ClO}_{4}\right), 1293\left(\mathrm{M}^{+}\right)$. Anal. Calcd for $\mathrm{C}_{61} \mathrm{H}_{52} \mathrm{~N}_{4} \mathrm{O}_{8} \mathrm{Pt}_{2} \mathrm{Cl}_{2} \mathrm{P}_{2}$ : C, 49.10; H, 3.51; N, 3.75 . Found: $\mathrm{C}, 48.96 ; \mathrm{H}, 3.47 ; \mathrm{N}, 3.70 .{ }^{1} \mathrm{H}$ NMR (DMSO- $\left.d_{6}, 500 \mathrm{MHz}\right)$ : $\delta 1.44 ; 1.78 ; 2.84$ (broad multiplets, $\left.10 \mathrm{H}, \mathrm{P}\left(\mathrm{CH}_{2}\right)_{5} \mathrm{P}\right), 6.48$ (broad $\mathrm{s}$, $2 \mathrm{H}), 6.68$ (broad s, 2H), 6.98-7.06 (m, 4H), 7.25-7.52 (m, 18H), $7.69-7.81(\mathrm{~m}, 8 \mathrm{H}), 8.21-8.36(\mathrm{~m}, 6 \mathrm{H}), 8.52-8.55(\mathrm{~m}, 2 \mathrm{H}) .{ }^{13} \mathrm{C}\left\{{ }^{1} \mathrm{H}\right\}$ NMR (DMSO- $\left.d_{6}\right): \delta 24.1\left(\mathrm{~d},{ }^{1} J(\mathrm{PC})=37.8 \mathrm{~Hz}, \mathrm{PCH}_{2}\right), 25.0(\mathrm{~s}$, $\left.\mathrm{P}\left(\mathrm{CH}_{2}\right)_{2} \mathrm{CH}_{2}\right), 30.9-31.2\left(\mathrm{~m}, \mathrm{PCH}_{2} \mathrm{CH}_{2}\right), 120.3,124.7-136.8,141.3$, 142.9, 147.7, 150.9, 153.5, 158.0, 162.7. ${ }^{31} \mathrm{P}\left\{{ }^{1} \mathrm{H}\right\}$ NMR $\left(\mathrm{CD}_{3} \mathrm{CN}\right): \delta$ $19.53\left({ }^{1} J(\mathrm{PtP})=3972 \mathrm{~Hz}\right)$

X-ray Crystallography. Crystals of $\mathbf{4 b}\left(\mathrm{ClO}_{4}\right)_{2} \cdot 5 \mathrm{H}_{2} \mathrm{O}$ and $\mathbf{9}\left(\mathrm{PF}_{6}\right)$ were obtained by vapor diffusion of diethyl ether into acetonitrile solutions. Crystal data and details of data collection and refinement are summarized in Table 1. The following data are listed in the order $\mathbf{4 b}\left(\mathrm{ClO}_{4}\right)_{2} \cdot 5 \mathrm{H}_{2} \mathrm{O} / \mathbf{9}\left(\mathrm{PF}_{6}\right)$. A total of $10201 / 2841$ unique reflections were collected at $298 \mathrm{~K}$ on a Nonius diffractometer $(\lambda(\mathrm{Mo} \mathrm{K} \alpha)=0.7107$ $\AA, 2 \theta_{\max }=45 / 50^{\circ}$ ). The structure was solved by Patterson methods, expanded using Fourier techniques, and refined by least-squares treatment on $F^{2}$ using the NRCVAX program: $R=0.059 / 0.025, \mathrm{w} R$ $=0.058 / 0.027, \mathrm{GoF}=1.75 / 1.48$, for $3760 / 2257$ absorption-corrected (transmission $0.93-1.00 / 0.63-1.00$ ) reflections with $I>2 \sigma(I)$ and $847 /$ 229 parameters. The pairs of atoms $\mathrm{N}(1) / \mathrm{C}(16)$ and $\mathrm{N}(3) / \mathrm{C}(39)$ for $4 \mathbf{b}\left(\mathrm{ClO}_{4}\right)_{2} \cdot 5 \mathrm{H}_{2} \mathrm{O}$ and $\mathrm{N}(1) / \mathrm{C}(12)$ for $\mathbf{9}\left(\mathrm{PF}_{6}\right)$ were differentiated by their temperature factors; interchanging the respective $\mathrm{C}$ and $\mathrm{N}$ atoms resulted in unreasonable temperature factors and/or higher $R$ values. For $\mathbf{9}\left(\mathrm{PF}_{6}\right)$, the two halves of the cation are related by a 2 -fold axis through $\mathrm{C}(18)$. Primed atoms are located at $(-x, y, 1 / 2-z)$.

\section{Results and Discussion}

Synthesis and Characterization. The cyclometalating ligands HL $^{1-6}$ (Figure 2) are readily prepared by Kröhnke syntheses using the appropriate enone and 2-pyridacylpyridinium iodide in the presence of excess ammonium acetate. ${ }^{32}$ Complexes $1 \mathbf{1}-$ 6a are synthesized by modification of the procedure reported by Constable and co-workers. ${ }^{34}$ The coordinated chloride ligand in this series allows facile derivatization of the $\left[\mathrm{Pt}\left(\mathrm{L}^{1-6}\right)\right]$ moieties. The cationic derivative $\left[\mathrm{Pt}\left(\mathrm{L}^{1}\right) \mathrm{py}\right]^{+}(7)$ is afforded by reaction of $\mathbf{1 a}$ with pyridine at room temperature. 
<smiles>[R]c1cc(-c2ccccc2)nc(-c2ccccn2)c1</smiles>

$$
\begin{array}{r}
\mathrm{R}=\mathrm{H}, \mathrm{HL}^{1} \\
\mathrm{Ph}, \mathrm{HL}^{2} \\
\text { 4-ClPh, } \mathrm{HL}^{3} \\
\text { 4-MePh, } \mathrm{HL}^{4} \\
\text { 4-MeOPh, } \mathrm{HL}^{5} \\
\text { 3,4,5-(MeO) }{ }_{3} \mathrm{Ph} \mathrm{HL}^{6}
\end{array}
$$<smiles></smiles>

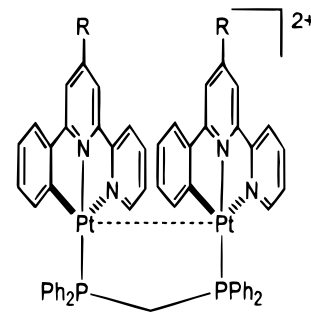

$\mathrm{Pt}\left(\mathrm{L}^{1-6}\right) \mathrm{Cl}, \mathbf{1} \mathbf{a}-\mathbf{6 a}$

$\left.\left[\mathrm{Pt}_{2}\left(\mathrm{~L}^{1-6}\right)\right)_{2}(\mu-\mathrm{dppm})\right]^{2+}, \mathbf{1 b}-\mathbf{6 b}$

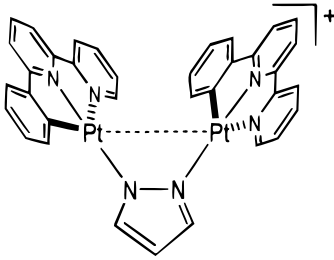

$\left[\mathrm{Pt}_{2}\left(\mathrm{~L}^{1}\right)_{2}(\mu-\mathrm{pz})\right]^{+}, 9$

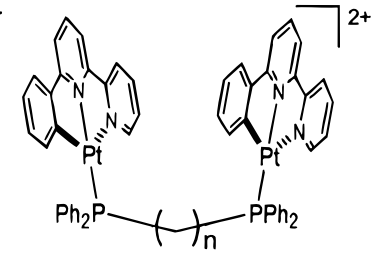

$\left[\mathrm{Pt}_{2}\left(\mathrm{~L}^{1}\right)_{2}\left(\mu-\mathrm{dpp} \mathrm{C}_{n}\right)\right]^{2+}$
Figure 2.

The binuclear complexes $\mathbf{1 b}-\mathbf{6 b}$ and $\mathbf{9 - 1 1}$ are conveniently obtained in moderate to high yields by treatment of the corresponding mononuclear precursor with the diphosphine or pyrazole (for 9). The ${ }^{31} \mathrm{P}\left\{{ }^{1} \mathrm{H}\right\}$ NMR spectra of the dicationic derivatives $\mathbf{1 b}-\mathbf{6 b}$ contain one signal with multiple ${ }^{195} \mathrm{Pt}$ satellites due to one- and three-bond couplings. These spectral patterns indicate the presence of a $\mathrm{Pt}_{2}(\mu-\mathrm{dppm})$ unit with chemically equivalent phosphorus atoms. ${ }^{35}$ In the ${ }^{1} \mathrm{H}$ NMR spectra, the dppm methylene group appears as a distinctive triplet resonance. The positive FAB mass spectra for $\mathbf{1 b}-\mathbf{6 b}$ contain signals corresponding to the $\left(\mathrm{M}^{+}+\mathrm{ClO}_{4}\right)$ fragment as well as the molecular cation $\mathrm{M}^{+}$.

Crystal Structures and $\mathbf{P t}-\mathbf{P t}$ Distances. The crystal structures of complex $\mathbf{4} \mathbf{b}$ bearing the tolyl group and the pyrazolate derivative 9 were determined by X-ray crystallography (Figures 3 and 4 respectively, Table 2). Since the structural parameters for $\mathbf{1 b}^{29}$ and $\mathbf{3} \mathbf{b}^{3 \mathrm{a}}$ have previously been elucidated, we can compare the effects of different substituents (H: 1b; 4-chlorophenyl: 3b; 4-tolyl: 4b) on 6-phenyl-2,2'bipyridine upon the solid-state and in particular $\pi-\pi$ stacking interactions in these complexes. As in $\mathbf{1} \mathbf{b}$ and $\mathbf{3 b}$, the platinum centers in $\mathbf{4 b}$ reside in distorted square planar environments and the molecular structure consists of two $\left[\mathrm{Pt}\left(\mathrm{L}^{4}\right)\right]$ units linked by a dppm bridge. The adjacent [Pt(6-phenyl-2,2'-bipyridine)] fragments are virtually parallel with a dihedral angle of $3.4^{\circ}$ (cf. $4.6^{\circ}$ in $\mathbf{3 b}$ and $6.1^{\circ}$ in $\mathbf{1 b}$ ). The intramolecular $\mathrm{Pt}-\mathrm{Pt}$ contacts for $\mathbf{1 b}, \mathbf{3 b}$ and $\mathbf{4 b}$ (3.270(1), 3.150(1), and 3.245(2) $\AA$, respectively) are slightly longer than those in the related derivatives $\left[\mathrm{Pt}_{2}(\operatorname{tpy})_{2}(\mu-\mathrm{L})\right]^{3+}\left(\mathrm{L}=\right.$ anion of guanidine ${ }^{25}$ and canavanine: ${ }^{26}$ mean 3.081 and $2.988 \AA$, respectively), but they nevertheless fall within the range of intermetal distances (3.09$3.50 \AA$ A) observed in monomeric Pt(II) extended linear-chain structures. ${ }^{11 \mathrm{a}}$

(35) Langrick, C. R.; McEwan, D. M.; Pringle, P. G.; Shaw, B. L. J. Chem. Soc., Dalton Trans. 1983, 2487.

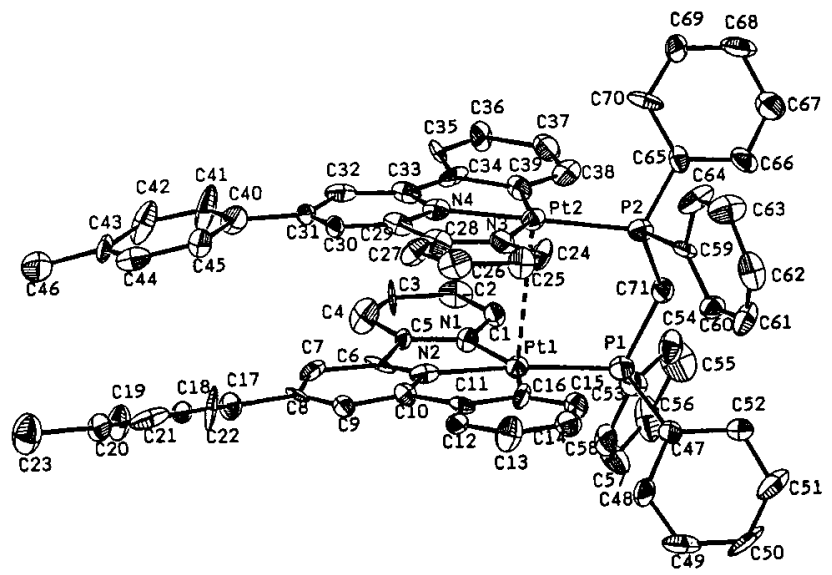

Figure 3. Perspective view of $\left[\mathrm{Pt}_{2}\left(\mathrm{~L}^{4}\right)_{2}(\mu \text {-dppm) }]^{2+}(\mathbf{4 b}, 30 \%\right.$ probability ellipsoids).

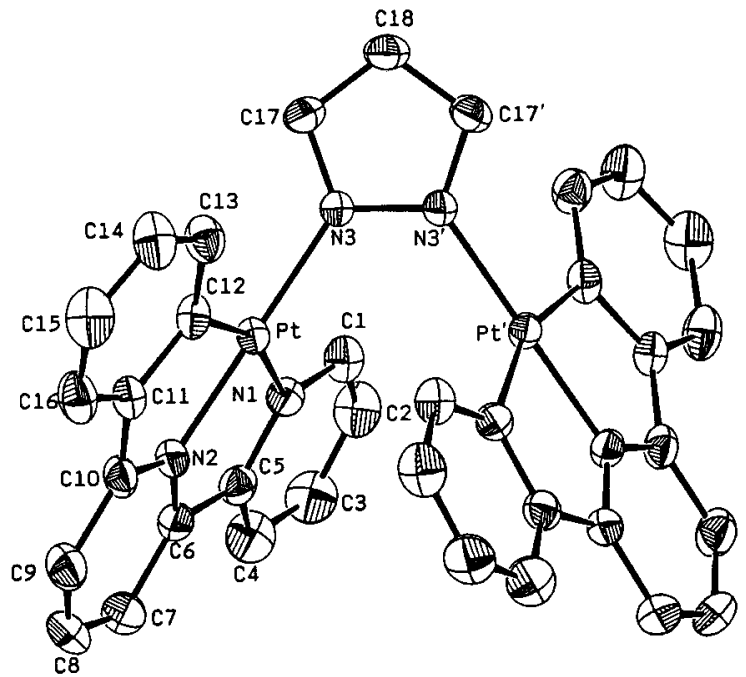

Figure 4. Perspective view of $\left[\mathrm{Pt}_{2}\left(\mathrm{~L}^{1}\right)_{2}(\mu-\mathrm{pz})\right]^{+}(\mathbf{9}, 40 \%$ probability ellipsoids). The two halves of the cation are related by a 2 -fold axis through $\mathrm{C}(18)$.

Table 2. Selected Bond Lengths ( $\mathrm{A})$ and Angles (deg)

\begin{tabular}{lccr}
\hline & Complex & $4 \mathbf{b}\left(\mathrm{ClO}_{4}\right)_{2} \cdot 5 \mathrm{H}_{2} \mathrm{O}$ & \\
$\mathrm{Pt}(1)-\mathrm{Pt}(2)$ & $3.245(2)$ & $\mathrm{Pt}(2)-\mathrm{P}(2)$ & $2.241(8)$ \\
$\mathrm{Pt}(1)-\mathrm{P}(1)$ & $2.251(8)$ & $\mathrm{Pt}(2)-\mathrm{N}(3)$ & $2.13(2)$ \\
$\mathrm{Pt}(1)-\mathrm{N}(1)$ & $2.17(2)$ & $\mathrm{Pt}(2)-\mathrm{N}(4)$ & $2.01(2)$ \\
$\mathrm{Pt}(1)-\mathrm{N}(2)$ & $1.99(2)$ & $\mathrm{Pt}(2)-\mathrm{C}(39)$ & $1.94(3)$ \\
$\mathrm{Pt}(1)-\mathrm{C}(16)$ & $2.04(3)$ & $\mathrm{P}(2)-\mathrm{C}(71)$ & $1.79(3)$ \\
$\mathrm{Pt}(2)-\mathrm{Pt}(1)-\mathrm{P}(1)$ & $89.6(2)$ & $\mathrm{Pt}(1)-\mathrm{P}(1)-\mathrm{C}(71)$ & $113.3(9)$ \\
$\mathrm{Pt}(2)-\mathrm{Pt}(1)-\mathrm{N}(1)$ & $94.1(5)$ & $\mathrm{P}(1)-\mathrm{Pt}(1)-\mathrm{N}(1)$ & $104.0(5)$ \\
$\mathrm{Pt}(2)-\mathrm{Pt}(1)-\mathrm{N}(2)$ & $94.8(5)$ & $\mathrm{P}(1)-\mathrm{Pt}(1)-\mathrm{N}(2)$ & $175.2(6)$ \\
$\mathrm{Pt}(2)-\mathrm{Pt}(1)-\mathrm{C}(16)$ & $88.4(7)$ & $\mathrm{P}(1)-\mathrm{Pt}(1)-\mathrm{C}(16)$ & $101.2(7)$ \\
$\mathrm{Pt}(1)-\mathrm{Pt}(2)-\mathrm{P}(2)$ & $89.3(2)$ & $\mathrm{Pt}(2)-\mathrm{P}(2)-\mathrm{C}(71)$ & $111.5(9)$ \\
$\mathrm{Pt}(1)-\mathrm{Pt}(2)-\mathrm{N}(3)$ & $93.4(6)$ & $\mathrm{P}(2)-\mathrm{Pt}(2)-\mathrm{N}(3)$ & $109.8(6)$ \\
$\mathrm{Pt}(1)-\mathrm{Pt}(2)-\mathrm{N}(4)$ & $89.4(5)$ & $\mathrm{P}(2)-\mathrm{Pt}(2)-\mathrm{N}(4)$ & $176.0(6)$ \\
$\mathrm{Pt}(1)-\mathrm{Pt}(2)-\mathrm{C}(39)$ & $90.2(8)$ & $\mathrm{P}(2)-\mathrm{Pt}(2)-\mathrm{C}(39)$ & $96.9(8)$ \\
& & & \\
& $\left.\mathrm{Complex} 9(\mathrm{PF})_{6}\right)$ & $1.368(8)$ \\
$\mathrm{Pt}-\mathrm{N}(1)$ & $2.113(5)$ & $\mathrm{N}(3)-\mathrm{N}\left(3^{\prime}\right)$ & $1.340(8)$ \\
$\mathrm{Pt}-\mathrm{N}(2)$ & $1.959(5)$ & $\mathrm{N}(3)-\mathrm{C}(17)$ & $1.384(9)$ \\
$\mathrm{Pt}-\mathrm{N}(3)$ & $2.009(4)$ & $\mathrm{C}(17)-\mathrm{C}(18)$ & \\
$\mathrm{Pt}-\mathrm{C}(12)$ & $1.999(6)$ & $\mathrm{Pt}-\mathrm{Pt}{ }^{\prime}$ & \\
$\mathrm{N}(1)-\mathrm{Pt}-\mathrm{N}(2)$ & $79.3(2)$ & $\mathrm{N}(3)-\mathrm{Pt}-\mathrm{C}(12)$ & $96.2(2)$ \\
$\mathrm{N}(1)-\mathrm{Pt}-\mathrm{N}(3)$ & $102.4(2)$ & $\mathrm{Pt}-\mathrm{N}(3)-\mathrm{N}\left(3^{\prime}\right)$ & $123.6(3)$ \\
$\mathrm{N}(1)-\mathrm{Pt}-\mathrm{C}(12)$ & $161.4(2)$ & $\mathrm{N}(3)-\mathrm{N}\left(3^{\prime}\right)-\mathrm{C}(17)$ & $108.3(5)$
\end{tabular}

The distinct difference between these structures is the torsion angle about the $\mathrm{Pt}-\mathrm{Pt}$ axis, which is $44.6,27.2$, and $20.7^{\circ}$ for $\mathbf{1 b}, \mathbf{3 b}$, and $\mathbf{4 b}$ (defined by the angle between the $\mathrm{Pt}(1)-$ 
Table 3. UV-Visible Spectral Data for Complexes 1a-6a, 7, and $\mathbf{8}^{a}$

\begin{tabular}{cc}
\hline complex & $\lambda_{\max } / \mathrm{nm}\left(\epsilon / \mathrm{dm}^{3} \mathrm{~mol}^{-1} \mathrm{~cm}^{-1}\right)$ \\
\hline$\left[\mathrm{Pt}\left(\mathrm{L}^{1}\right) \mathrm{Cl}\right], \mathbf{1 a}$ & $278(19600), 325(9500), 360(5000)$, \\
& $430(1550), 510(180)$ \\
{$\left[\mathrm{Pt}\left(\mathrm{L}^{2}\right) \mathrm{Cl}\right], \mathbf{2 a}$} & $285(35000), 334(16100), 369(7600)$, \\
& $435(3100), 519(150)$ \\
{$\left[\mathrm{Pt}\left(\mathrm{L}^{3}\right) \mathrm{Cl}\right], \mathbf{3 a}$} & $288(20000), 332(7800), 369(4000)$, \\
& $434(1950), 523(260)$ \\
{$\left[\mathrm{Pt}\left(\mathrm{L}^{4}\right) \mathrm{Cl}\right], \mathbf{4 a}$} & $292(34000), 334(17800), 368(7800)$, \\
& $438(3500), 522(200)$ \\
{$\left[\mathrm{Pt}\left(\mathrm{L}^{5}\right) \mathrm{Cl}\right], \mathbf{5 a}$} & $278(21800), 314(18700) 340(17100)$, \\
{$\left[\mathrm{Pt}\left(\mathrm{L}^{6}\right) \mathrm{Cl}\right], \mathbf{6 a}$} & $437(3300), 522(540)$ \\
& $278(29700), 336(19200), 418(5000)$, \\
{$\left[\mathrm{Pt}\left(\mathrm{L}^{1}\right) \mathrm{py}\right] \mathrm{ClO}$} & $437(5200), 525(570)$ \\
$\mathbf{7}(\mathrm{ClO})_{4}{ }^{b}$ & $259(37500), 335(16000) 350(15700)$, \\
{$\left[\mathrm{Pt}\left(\mathrm{L}^{1}\right) \mathrm{PPh}_{3}\right] \mathrm{ClO}{ }_{4}$,} & $422(590), 498(90)$ \\
$\mathbf{8}(\mathrm{ClO})^{b}$ & $266(24000), 336(11200), 350(10700)$, \\
${ }^{a} \mathrm{Measured}^{b}$ at room temperature in $\mathrm{CH}_{2} \mathrm{Cl}_{2}$ unless otherwise stated. \\
${ }^{b}$ In acetonitrile.
\end{tabular}

${ }^{b}$ In acetonitrile.

$\mathrm{Pt}(2)-\mathrm{N}(2)$ and $\mathrm{Pt}(1)-\mathrm{Pt}(2)-\mathrm{N}(4)$ planes $)$, respectively. The orientation in $\mathbf{1 b}$ optimizes the $\pi$-stacking interactions of the aromatic ligand $\mathrm{L}^{1}$, since face-to-face overlap of $\pi$ orbitals in an eclipsed manner (i.e. torsion angle ca. $0^{\circ}$ ) introduces repulsive forces. ${ }^{36}$ The reduced torsion angles in $\mathbf{3 b}$ and $\mathbf{4 b}$ are presumably manifestations of further $\pi-\pi$ interactions between the respective 4-chlorophenyl and tolyl substituents in achieving the most stable conformation. In addition, these partially staggered geometry essentially eliminate steric repulsion between the chloro and methyl groups in $\mathbf{3 b}$ and $\mathbf{4 b}$, respectively. We therefore attribute the varying torsion angles in these structures to the different geometrical demands for $\pi$-stacking interactions by the cyclometalating ligands $\mathrm{L}^{1}, \mathrm{~L}^{3}$ and $\mathrm{L}^{4}$.

The platinum centers in the structure of complex $\mathbf{9}$ are in distorted square planar geometry, but unlike in the dppm derivatives, the $\left[\mathrm{Pt}\left(\mathrm{L}^{1}\right)\right]$ moieties are not parallel due to the rigid coordination mode of the pyrazolate linker (Figure 4). The comparable bond lengths within the pz ring (range 1.340(8)1.384(9) $\AA$ ) is consistent with the expected $\pi$-delocalization. The resultant $\mathrm{Pt}-\mathrm{Pt}$ distance of 3.612(2) $\AA$ is significantly longer than that in $\left[\mathrm{Pt}_{2}(\mathrm{tpy})_{2}(\mu-\mathrm{pz})\right]\left(\mathrm{ClO}_{4}\right)_{3}(3.432(1) \AA)^{24}$ and implies negligible metal-metal communication, although weak $\pi-\pi$ interactions are possible. The effect of this and the general consequences of longer metal - metal and $\pi-\pi$ separations upon the excited-state properties of these binuclear complexes will be discussed later. Close intermolecular contacts are not observed in the crystal lattice of $\mathbf{4 b}$ and $\mathbf{9}$.

Absorption and Emission Spectroscopy. Mononuclear Complexes. The UV-visible spectral data of the monomeric complexes $\left[\mathrm{Pt}\left(\mathrm{L}^{1-6}\right) \mathrm{Cl}\right](\mathbf{1 a}-\mathbf{6 a}),\left[\mathrm{Pt}\left(\mathrm{L}^{1}\right) \mathrm{py}\right]^{+}(\mathbf{7})$, and $\left[\mathrm{Pt}\left(\mathrm{L}^{1}\right)-\right.$ $\left.\mathrm{PPh}_{3}\right]^{+}(\mathbf{8})$ are listed in Table 3. Their absorption spectra obey Beer's law in the concentration range $10^{-6}-10^{-4} \mathrm{M}$. The optical spectrum of $1 \mathbf{a}$ has been described previously. ${ }^{29}$ Similarly, the high-energy region $(\lambda<370 \mathrm{~nm})$ in the absorption spectra of $\mathbf{2 a}-\mathbf{6 a}$, with 4-aryl substituents on the 6-phenyl-2,2'-bipyridine ligand, is dominated by ${ }^{1} \mathrm{IL}\left(\pi \rightarrow \pi^{*}\right)$ transitions, while the moderately intense low-energy bands with $\lambda_{\max }$ in the range 434-438 $\mathrm{nm}$ are assigned to ${ }^{1} \mathrm{MLCT}(5 \mathrm{~d}) \mathrm{Pt} \rightarrow \pi^{*}(\mathrm{~L})$ transitions (see Figure 5 for $\mathbf{6 a}$ ). The weak absorption tails at 519-525 $\mathrm{nm}\left(\epsilon<600 \mathrm{dm}^{3} \mathrm{~mol}^{-1} \mathrm{~cm}^{-1}\right)$ are attributed to ${ }^{3} \mathrm{MLCT}$ transition.

The monomeric cyclometalated platinum(II) complexes studied in this work are emissive in solution and solid states (Tables

(36) Hunter, C. A.; Sanders, J. K. M. J. Am. Chem. Soc. 1990, 112, 5525.

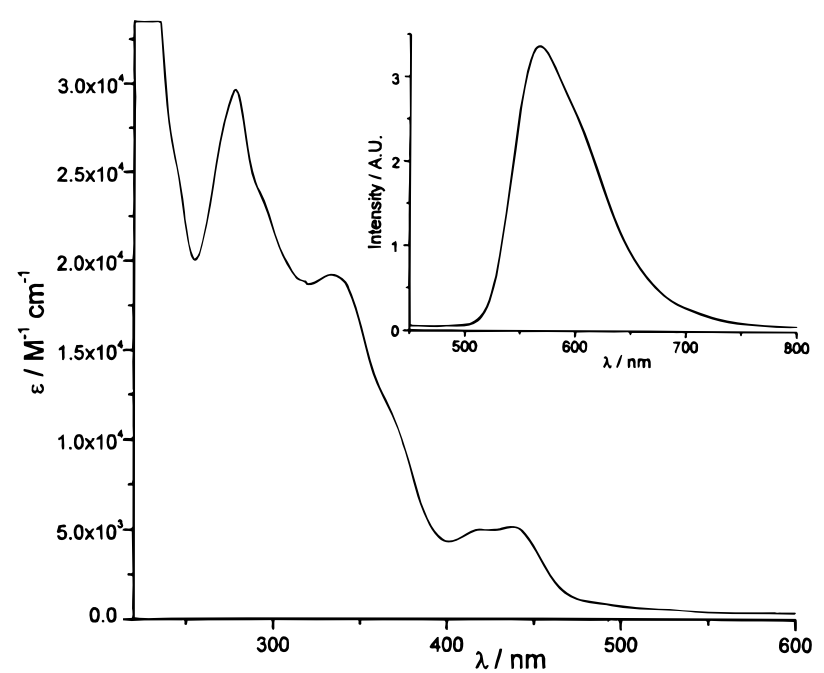

Figure 5. UV-vis absorption and emission (inset: $\lambda_{\mathrm{ex}} 350 \mathrm{~nm}$ ) spectra of $6 \mathbf{a}$ in $\mathrm{CH}_{2} \mathrm{Cl}_{2}$ at $298 \mathrm{~K}$.

Table 4. Solution Emission Data for Complexes 1a-6a, 7, and $\mathbf{8}^{a}$

\begin{tabular}{ccc}
\hline complex & $\begin{array}{c}298 \mathrm{~K} \text { data: } \lambda_{\max } / \mathrm{nm} ; \\
\tau_{\mathrm{o}} / \mu \mathrm{s} ; \phi_{\mathrm{o}} ; k_{\mathrm{q}} / \mathrm{M}^{-1} \mathrm{~s}^{-1}\end{array}$ & $\begin{array}{c}77 \mathrm{~K} \mathrm{data}: \\
\lambda_{\max } / \mathrm{nm}\end{array}$ \\
\hline$\left[\mathrm{Pt}\left(\mathrm{L}^{1}\right) \mathrm{Cl}\right], \mathbf{1 a}$ & $565 ; 0.51 ; 0.025 ; 3.1 \times 10^{9}$ & $535,567(\max )$, \\
& & 610,600 \\
{$\left[\mathrm{Pt}\left(\mathrm{L}^{2}\right) \mathrm{Cl}\right], \mathbf{2 a}$} & $564 ; 0.60 ; 0.052 ; 4.1 \times 10^{9}$ & $555(\max ), 592$ \\
{$\left[\mathrm{Pt}\left(\mathrm{L}^{3}\right) \mathrm{Cl}\right], \mathbf{3 a}$} & $568 ; 0.52 ; 0.054 ; 2.4 \times 10^{9}$ & $572(\max ), 595$ \\
{$\left[\mathrm{Pt}\left(\mathrm{L}^{4}\right) \mathrm{Cl}\right], \mathbf{4 a}$} & $562 ; 0.62 ; 0.064 ; 2.2 \times 10^{9}$ & $575(\max ), 623$ \\
{$\left[\mathrm{Pt}\left(\mathrm{L}^{5}\right) \mathrm{Cl}\right], \mathbf{5 a}$} & $563 ; 0.72 ; 0.068 ; 2.9 \times 10^{9}$ & $578(\max ), 613$ \\
{$\left[\mathrm{Pt}\left(\mathrm{L}^{6}\right) \mathrm{Cl}\right], \mathbf{6 a}$} & $566 ; 0.63 ; 0.057 ; 1.6 \times 10^{9}$ & $552(\max ), 589$ \\
{$\left[\mathrm{Pt}\left(\mathrm{L}^{1}\right) \mathrm{py}\right] \mathrm{ClO}_{4}$,} & $540 ; 1.47 ; 0.066 ; 1.5 \times 10^{9}$ & $522(\max ), 554^{b}$ \\
$7\left(\mathrm{ClO}_{4}\right)$ & & \\
{$\left[\mathrm{Pt}\left(\mathrm{L}^{1}\right) \mathrm{PPh}_{3}\right] \mathrm{ClO}_{4}$,} & $535 ; 0.89 ; 0.062 ; 3.2 \times 10^{8}$ & $527(\max ), 560^{b}$ \\
$\mathbf{8}\left(\mathrm{ClO}_{4}\right)$ & &
\end{tabular}

${ }^{a}$ Complex concentration $1 \times 10^{-5} \mathrm{M}$, in $\mathrm{CH}_{2} \mathrm{Cl}_{2}$ unless otherwise stated; $1 / \tau=k_{\mathrm{obs}}=k_{\mathrm{q}}[$ complex $]+k_{\mathrm{o}} \cdot{ }^{b}$ In acetonitrile.

Table 5. Solid-State Emission Data for Complexes 1a-6a, 7, and 8

\begin{tabular}{cll}
\hline \multicolumn{1}{c}{ complex } & \multicolumn{1}{c}{$\begin{array}{c}298 \mathrm{~K} \text { data: } \\
\lambda_{\max } / \mathrm{nm}\left(\tau_{\mathrm{o}} / \mu \mathrm{s}\right)\end{array}$} & \multicolumn{1}{c}{$\begin{array}{c}77 \mathrm{~K} \mathrm{data} \\
\lambda_{\max } / \mathrm{nm}\end{array}$} \\
\hline$\left[\mathrm{Pt}\left(\mathrm{L}^{1}\right) \mathrm{Cl}\right], \mathbf{1 a}$ & $665(0.40)$ & 700 \\
{$\left[\mathrm{Pt}\left(\mathrm{L}^{2}\right) \mathrm{Cl}\right], \mathbf{2 a}$} & $603(\max ), 646(0.66)$ & $597(\max ), 643,702$ \\
{$\left[\mathrm{Pt}\left(\mathrm{L}^{3}\right) \mathrm{Cl}\right], \mathbf{3 a}$} & $600(\max ), 640(0.27)$ & $594(\max ), 643,700$ \\
{$\left[\mathrm{Pt}\left(\mathrm{L}^{4}\right) \mathrm{Cl}\right], \mathbf{4 a}$} & $602(\max ), 646(0.51)$ & $591(\max ), 637,700$ \\
{$\left[\mathrm{Pt}\left(\mathrm{L}^{5}\right) \mathrm{Cl}\right], \mathbf{5 a}$} & $676(0.34)$ & 716 \\
{$\left[\mathrm{Pt}\left(\mathrm{L}^{6}\right) \mathrm{Cl}\right], \mathbf{6 a}$} & $687(0.25)$ & 722 \\
{$\left[\mathrm{Pt}\left(\mathrm{L}^{1}\right) \mathrm{py}^{2} \mathrm{ClO}_{4}\right.$,} & $578(\max ), 615(1.80)$ & $572(\max ), 611$ \\
$\quad \mathbf{7}\left(\mathrm{ClO}_{4}\right)$ & & \\
{$\left[\mathrm{Pt}\left(\mathrm{L}^{1}\right) \mathrm{PPh}_{3}\right] \mathrm{ClO}_{4}$,} & $600(2.41)$ & $550(\max ), 570$ \\
$\mathbf{8}\left(\mathrm{ClO}_{4}\right)$ & &
\end{tabular}

4 and 5, respectively). With reference to earlier work, the structureless emissions of complexes $\mathbf{2 a - 6 a}$ in $\mathrm{CH}_{2} \mathrm{Cl}_{2}$ at room temperature are assigned as ${ }^{3} \mathrm{MLCT}$ in nature (see Figure 5 for 6a). Variation of the emission maxima is small (range 562$568 \mathrm{~nm}$ ), and no trends are apparent for the different parasubstituents. Hence there appears to be limited electronic communication between the 4-aryl group and the planar 6-phenyl-2,2'-bipyridine moiety. Minor solvatochromic effects are observed for $\mathbf{2} \mathbf{a}-\mathbf{6 a}$ : for example, the room-temperature emission of $\mathbf{2 a}$ shifts from $560 \mathrm{~nm}$ in acetonitrile to $567 \mathrm{~nm}$ in dichloromethane. The blue-shifted emission maxima for the cationic complexes $\mathbf{7}$ and $\mathbf{8}$ correlates with the greater charge on the $\mathrm{Pt}(\mathrm{II})$ center, which is expected to increase the energy of the ${ }^{3}$ MLCT transition.

The orange to red colors of $\mathbf{1 a - 6 a}$ in the solid state are noteworthy since these compounds absorb weakly at wave- 
Table 6. UV-Visible Spectral Data for Complexes $\mathbf{1 b}-\mathbf{6 b}$ and 9-11 in Acetonitrile

complex

$\left[\mathrm{Pt}_{2}\left(\mathrm{~L}^{1}\right)_{2}(\mu\right.$-dppm $\left.)\right]\left(\mathrm{ClO}_{4}\right)_{2}, \mathbf{1 b}\left(\mathrm{ClO}_{4}\right)_{2}$ $\left[\mathrm{Pt}_{2}\left(\mathrm{~L}^{2}\right)_{2}(\mu\right.$-dppm) $]\left(\mathrm{ClO}_{4}\right)_{2}, \mathbf{2 b}\left(\mathrm{ClO}_{4}\right)_{2}$ $\left[\mathrm{Pt}_{2}\left(\mathrm{~L}^{3}\right)_{2}(\mu\right.$-dppm $\left.)\right]\left(\mathrm{ClO}_{4}\right)_{2}, \mathbf{3 b}\left(\mathrm{ClO}_{4}\right)_{2}$ $\left[\mathrm{Pt}_{2}\left(\mathrm{~L}^{4}\right)_{2}(\mu\right.$-dppm $\left.)\right]\left(\mathrm{ClO}_{4}\right)_{2}, \mathbf{4 b}\left(\mathrm{ClO}_{4}\right)_{2}$ $\left[\mathrm{Pt}_{2}\left(\mathrm{~L}^{5}\right)_{2}(\mu\right.$-dppm $\left.)\right]\left(\mathrm{ClO}_{4}\right)_{2}, \mathbf{5 b}\left(\mathrm{ClO}_{4}\right)_{2}$ $\left[\mathrm{Pt}_{2}\left(\mathrm{~L}^{6}\right)_{2}(\mu\right.$-dppm) $]\left(\mathrm{ClO}_{4}\right)_{2}, \mathbf{6} \mathbf{b}\left(\mathrm{ClO}_{4}\right)_{2}$ $\left[\mathrm{Pt}_{2}\left(\mathrm{~L}^{1}\right)_{2}(\mu-\mathrm{pz})\right]\left(\mathrm{ClO}_{4}\right), \mathbf{9}\left(\mathrm{ClO}_{4}\right)$

$\left[\mathrm{Pt}_{2}\left(\mathrm{~L}^{1}\right)_{2}\left(\mu\right.\right.$-dppC $\left.\left.\mathrm{C}_{3}\right)\right]\left(\mathrm{ClO}_{4}\right)_{2}, \mathbf{1 0}\left(\mathrm{ClO}_{4}\right)_{2}$ $\left[\mathrm{Pt}_{2}\left(\mathrm{~L}^{1}\right)_{2}\left(\mu-\mathrm{dppC}_{5}\right)\right]\left(\mathrm{ClO}_{4}\right)_{2}, \mathbf{1 1}\left(\mathrm{ClO}_{4}\right)_{2}$ $\lambda_{\max } / \mathrm{nm}\left(\epsilon / \mathrm{dm}^{3} \mathrm{~mol}^{-1} \mathrm{~cm}^{-1}\right)$

340 (16 500), 420 (3900), 478 (sh, 2550), 504 (sh, 1900)

286 (52 400), 316 (39 600), 421 (4000), 481 (sh, 2700), 511 (sh, 1800)

293 (47 900), 320 (41 200), 420 (3900), 488 (sh, 2600), 515 (sh, 1850)

292 (45 400), 325 (45 300), 420 (4500), 477 (sh, 2650), 510 (sh, 1700)

345 (46 200), 482 (2600), 510 (sh, 1750)

346 (38 700), 485 (2600), 522 (sh, 1450)

266 (34 800), 323 (13 300), 356 (9700), 409 (2500), 510 (sh, 250)

336 (17 000), 350 (15 200), 426 (900), 494 (150)

334 (18 800), 350 (17 500), 422 (850), 494 (120) lengths longer than $450 \mathrm{~nm}$ in solution. Their intense colors are attributed to the propensity of these square planar $\mathrm{Pt}(\mathrm{II})$ species, like established diimine relatives, to undergo solid-state intermolecular metal-metal and ligand-ligand interactions which yield low-energy [d $\sigma^{*} \rightarrow \pi^{*}$ ] transitions (Figure 1). ${ }^{12}$ Structurally determined cyclometalated analogues exhibit $\mathrm{Pt}-$ Pt distances in the range 3.28-3.37 $\AA$ (for $\left[\mathrm{Pt}\left(\mathrm{L}^{1}\right)\left(\mathrm{CH}_{3} \mathrm{CN}\right)\right]$ $\mathrm{PF}_{6}{ }^{34}$ and $\left[\mathrm{Pt}(\mathrm{dpphen})\left(\mathrm{CH}_{3} \mathrm{CN}\right)\right] \mathrm{ClO}_{4}{ }^{27}$ (Hdpphen = 2,9-diphenyl1,10-phenanthroline), respectively) and $\pi-\pi$ separations of around $3.35 \AA$ (for $\left[\mathrm{Pt}\left(\mathrm{L}^{1}\right) \mathrm{PPh}_{3}\right] \mathrm{ClO}_{4}{ }^{29}$ ). At room temperature, microcrystalline samples of complexes $\mathbf{1 a}, \mathbf{5 a}$, and $\mathbf{6 a}$ emit with $\lambda_{\max }$ at 665, 676, and $687 \mathrm{~nm}$, respectively (Table 5). Upon cooling of the samples to $77 \mathrm{~K}$, the bandwidths of the emissions reduce and the emission maxima shift to 700,716 , and 722 $\mathrm{nm}$, respectively. These data are comparable to those for the binuclear analogues $\mathbf{1 b}, \mathbf{5 b}$, and $\mathbf{6 b}$ which display metal-metal and/or ligand-ligand interactions (see later) and suggest that the solid-state emissions of $\mathbf{1 a}, \mathbf{5 a}$, and $\mathbf{6} \mathbf{a}$ are ${ }^{3}\left[\mathrm{~d} \sigma^{*}, \pi^{*}\right]$ in nature. The red-shift for these solid-state emissions upon cooling can be rationalized by shortening of intermolecular PtPt and $\pi-\pi$ separations in the crystal lattice, which results in ${ }^{3}\left[\mathrm{~d} \sigma^{*}, \pi^{*}\right]$ emissions of lower energy. In contrast, complexes $\mathbf{2 a}-\mathbf{4 a}$ and $\mathbf{7}$ show a structured band at $298 \mathrm{~K}$ with emission maxima at significantly higher energies $\left(\lambda_{\max } 578-\right.$ $603 \mathrm{~nm}$ ) than the ${ }^{3}\left[\mathrm{~d} \sigma^{*}, \pi^{*}\right]$ emissions, while a blue shift is detected at $77 \mathrm{~K} ;{ }^{3} \mathrm{MLCT}$ excited states are therefore tentatively assigned.

Self-Quenching and 77 K Frozen Emission. Like planar aromatic systems, a small number of square planar platinum(II) complexes are known to display low-energy excimeric emission in concentrated fluid solutions ${ }^{20-22}\left(\mathrm{eq} 1, \mathrm{~N}^{\mathrm{N}}=\right.$

$\left[\mathrm{Pt}\left(\mathrm{N}^{\mathrm{N}}\right)(\mathrm{CN})_{2}\right]^{*}+\left[\mathrm{Pt}(\hat{\mathrm{N}} \mathrm{N})(\mathrm{CN})_{2}\right] \rightarrow$

$$
\left[\mathrm{Pt}\left(\mathrm{N}^{-} \mathrm{N}\right)(\mathrm{CN})_{2}\right]_{2}^{*}
$$

4,7-diphenyl-1,10-phenanthroline, 4,4'-di-tert-butyl-2,2'-bipyridine).

Self-quenching of the ${ }^{3}$ MLCT emission has been detected for complexes $\mathbf{1 a}-\mathbf{6 a}, \mathbf{7}$, and $\mathbf{8}$ at $298 \mathrm{~K}$ in $\mathrm{CH}_{2} \mathrm{Cl}_{2}$ (Table 4), but no excimeric emission with complex concentration up to $10^{-3} \mathrm{M}$ has been observed. In each case, a linear plot of $1 / \tau$ against complex concentration was produced. The intrinsic luminescence lifetimes $\tau_{\mathrm{o}}$ range from 0.51 to $1.47 \mu \mathrm{s}$, and selfquenching rate constants $k_{\mathrm{q}}$ of $3.2 \times 10^{8}$ (for $\mathrm{PPh}_{3}$ complex 8) to $4.1 \times 10^{9} \mathrm{dm}^{3} \mathrm{~mol}^{-1} \mathrm{~s}^{-1}$ are observed. The emission quantum yields $\phi_{0}$ of the substituted complexes $\mathbf{2 a - 6 a}$ range from 0.052 to 0.068 and are higher than that for $\mathbf{1 a}\left(\phi_{0} 0.025\right)$. This is reminiscent of studies on phenyl-substituted tpy complexes of ruthenium, where the increased quantum yield was ascribed to extended conjugation which stabilizes the emissive MLCT excited state relative to a radiationless $\mathrm{d}-\mathrm{d}$ transition. ${ }^{37}$

(37) Hecker, C. R.; Gushurst, A. K. I.; McMillin, D. R. Inorg. Chem. 1991, 30,538 .

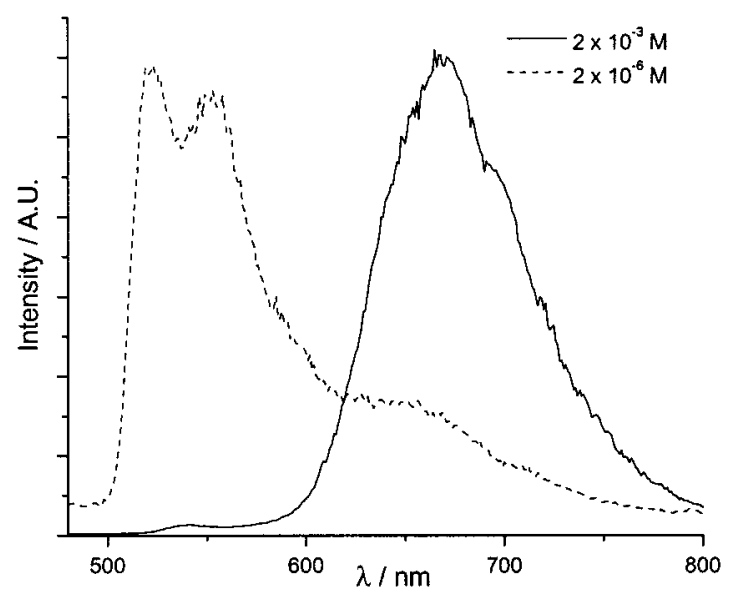

Figure 6. Normalized emission spectra of $\mathbf{7}$ at different concentrations in acetonitrile at $77 \mathrm{~K}\left(\lambda_{\mathrm{ex}} 350 \mathrm{~nm}\right)$.

The emissions of $\mathbf{1 a}-\mathbf{6 a}, \mathbf{7}$, and $\mathbf{8}$ at $77 \mathrm{~K}$ in frozen dichloromethane or acetonitrile have been studied (Table 4). Except for 7, their emissions are insensitive to the complex concentration in the range $10^{-6}-10^{-3} \mathrm{M}$. They are highly structured, with the most intense vibronic component being $v^{\prime}$ $=0$ to $v^{\prime \prime}=0$, which is characteristic of ${ }^{3}$ MLCT emissions.

The concentration-dependent emissive behavior of 7 in acetonitrile at $77 \mathrm{~K}$ has been investigated (Figure 6). At low concentrations $\left(\sim 10^{-6} \mathrm{M}\right)$, vibronic yellow emission with peak maxima at 522 and $554 \mathrm{~nm}$ (vibronic progression $1110 \mathrm{~cm}^{-1}$ ) is observed. Increasing the complex concentration yielded a new red emission centered at $670 \mathrm{~nm}$ at the expense of the yellow band. Indeed, at complex concentration $>10^{-3} \mathrm{M}$, the highenergy emission is completely replaced by the low-energy band at $670 \mathrm{~nm}$. We note that the UV-vis absorption spectrum of 7 in acetonitrile at $298 \mathrm{~K}$ follows Beer's law for concentration $\leq$ $10^{-3} \mathrm{M}$; hence, no ground-state oligomerization is evident at 298 K. Emission from solid-state phases in frozen acetonitrile is discounted because this occurs at $\lambda_{\max } 572$ and $611 \mathrm{~nm}$ at 77 $\mathrm{K}$ (Table 5). We suggest that, in frozen acetonitrile solution, the complex cations of $\mathbf{7}$ form weakly interacting dimeric pairs, and the $670 \mathrm{~nm}$ band can therefore be ascribed to a ${ }^{3}\left[\mathrm{~d} \sigma^{*}, \pi^{*}\right]$ excited state.

Binuclear Complexes. Our earlier report on the parent 6-phenyl-2,2'-bipyridine complex $\left[\mathrm{Pt}_{2}\left(\mathrm{~L}^{1}\right)_{2}(\mu \text {-dppm) }]^{2+}(\mathbf{1 b})\right.$ assigned the lowest energy absorption band at $420 \mathrm{~nm}$ in acetonitrile to a singlet $\left[\mathrm{d} \sigma^{*} \rightarrow \pi^{*}\right]$ transition, while the emission at $652 \mathrm{~nm}$ was ascribed to a triplet $\left[\mathrm{d} \sigma^{*}, \pi^{*}\right]$ excited state (the MMLCT notation was previously used). ${ }^{29}$ We now proceed to examine the effects upon the $\left[\mathrm{d} \sigma^{*}, \pi^{*}\right.$ ] excited state of (A) different aryl substituents at the 4-position of 6-phenyl-2,2'bipyridine and of (B) bidentate ligands with various bridging lengths and geometry.

(A) Complexes with the $\mu$-dppm Ligand: Effects of the 4-Aryl Substituent. The UV-vis absorption spectra of $\mathbf{2 b -}$ 


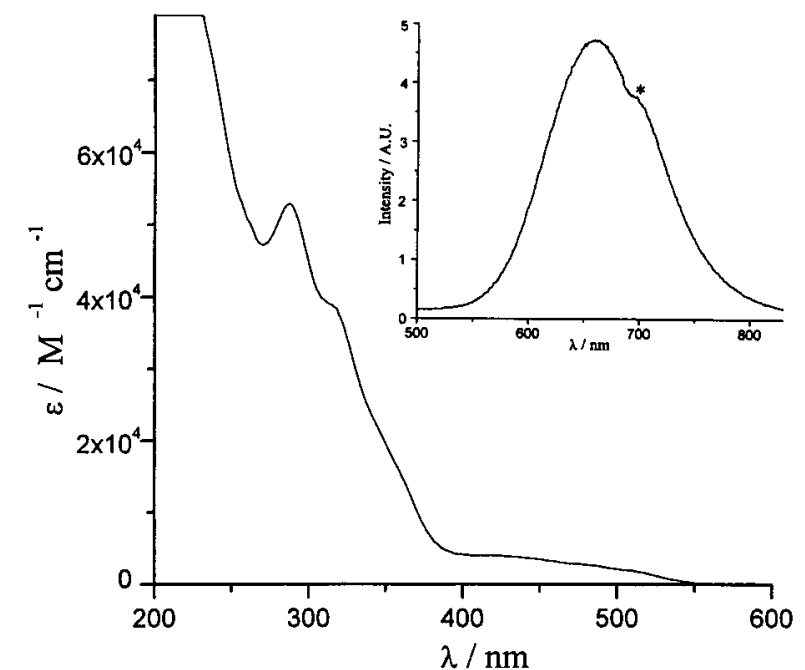

Figure 7. UV-vis absorption and emission (inset: $\lambda_{\mathrm{ex}} 350 \mathrm{~nm}, *$ denotes instrumental artifact) spectra of $\mathbf{2 b}$ in acetonitrile at $298 \mathrm{~K}$.

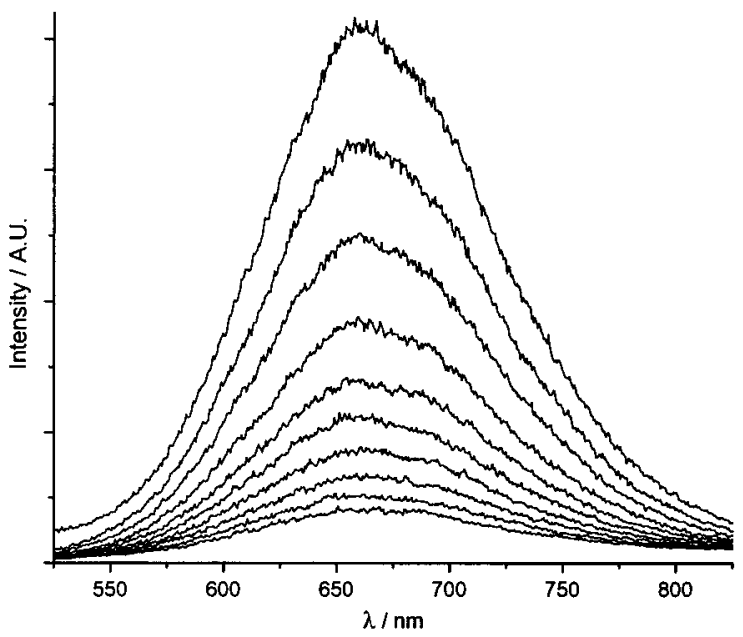

Figure 8. Time-resolved emission profile for $\mathbf{5 b}(0.01 \mathrm{mM}$ in acetonitrile) at $298 \mathrm{~K}$ (from top, 120, 200, 300, 400, 500, 600, 700, $\left.800,900,1000 \mathrm{~ns} ; \lambda_{\text {ex }} 350 \mathrm{~nm}\right)$.

$\mathbf{6 b}$ are generally similar to that of $\mathbf{1 b}$ (Table 6 , see Figure 7 for 2b). All binuclear complexes in this work are photoluminescent in solution (Table 7). Complexes $\mathbf{2 b}-\mathbf{6 b}$ exhibit structureless emissions with peak maxima at $654-662 \mathrm{~nm}$ in acetonitrile at room temperature, while at $77 \mathrm{~K}$ blue-shifted emissions are observed at $633-644 \mathrm{~nm}$. These are comparable to the emissive behavior of $\mathbf{1 b}$. Trends arising from the different nature of aryl substituents are not apparent. The time-resolved emission profiles for $\mathbf{2 b}-\mathbf{6 b}$ (Figure 8 for $\mathbf{5 b}$ ) each show a monoexponential decay, thus implying a single emitting species is present. The $77 \mathrm{~K}$ excitation spectrum of $\mathbf{5 b}$ in acetonitrile (monitoring emission at $640 \mathrm{~nm}$; see Supporting Information) corresponds with the absorption spectral bands, particularly in the 480-510 $\mathrm{nm}$ region (Table 6). In conjunction with the observed intramolecular $\mathrm{Pt}-\mathrm{Pt}$ contacts in $\mathbf{3 b}$ and $\mathbf{4 b}$, the emissions of $\mathbf{2} \mathbf{b}-\mathbf{6} \mathbf{b}$ are ascribed to ${ }^{3}\left[\mathrm{~d} \sigma^{*}, \pi^{*}\right]$ excited states such as for $\mathbf{1 b}$. Self-quenching of the emissions in acetonitrile is difficult to quantify accurately due to the relatively short lifetimes. In dichloromethane, self-quenching rate constants of $1.2 \times 10^{8}$ and $2.0 \times 10^{8} \mathrm{dm}^{3} \mathrm{~mol}^{-1} \mathrm{~s}^{-1}$ for $\mathbf{2 b}$ and $\mathbf{5 b}$ respectively were estimated, which are comparable with that for the mononuclear phosphine derivative $8\left(3.2 \times 10^{8} \mathrm{dm}^{3}\right.$ $\mathrm{mol}^{-1} \mathrm{~s}^{-1}$ ).

The solvatochromic behavior of the ${ }^{3}\left[\mathrm{~d} \sigma^{*}, \pi^{*}\right]$ emissions of $\mathbf{1} \mathbf{b}\left(\mathrm{ClO}_{4}\right)_{2}$ and $\mathbf{5} \mathbf{b}\left(\mathrm{ClO}_{4}\right)_{2}$ has been studied (Table 8). The respective emission energy, lifetime, and quantum yield are highly sensitive to the solvent polarity but insensitive to the complex concentration. For $\mathbf{5} \mathbf{b}\left(\mathrm{ClO}_{4}\right)_{2}$, the emission maximum shifts by $689 \mathrm{~cm}^{-1}$ from dichloromethane to dimethylformamide (DMF) while the luminescence lifetimes $\left(\tau_{\mathrm{o}}\right)$ and quantum yields $\left(\phi_{0}\right)$ change from 2.45 to $0.30 \mu$ s and 0.20 to less than 0.01 , respectively. This is ascribed to greater rates of non-radiative decay in highly polar solvents such as DMF. Significantly, these cyclometalated derivatives exhibit improved photophysical properties compared to the tpy congeners, which show very weak emission $\left(\phi_{0}\right.$ in order of $\left.10^{-4}\right)$ in solution at room temperature. $^{24,25}$

The solid-state emissions of $\mathbf{1 b}-\mathbf{6 b}$ (Table 9) are red-shifted in energy as the temperature is lowered to $77 \mathrm{~K}$. This may be attributed to the shortening of $\mathrm{Pt}-\mathrm{Pt}$ distances due to lattice contraction. The emissions of $\mathbf{1 b}, \mathbf{5 b}$, and $\mathbf{6} \mathbf{b}$ are similar to that for the mononuclear counterparts (see above), and we propose that they have the same electronic origin, namely ${ }^{3}\left[\mathrm{~d} \sigma^{*}, \pi^{*}\right]$.

(B) Complexes with the 6-Phenyl-2,2'-bipyridine $\left(\mathrm{L}^{1}\right)$ Ligand: Effects of the Bridging Ligand. To influence the distance and interaction between $\left[\mathrm{Pt}\left(\mathrm{L}^{1}\right)\right]$ moieties in a binuclear molecule, the pyrazolate derivative 9 and the $\mathrm{C}_{3}$ and $\mathrm{C}_{5}$ diphosphine complexes $\mathbf{1 0}$ and 11, respectively, were prepared. Consequences upon the excited-state properties can be probed by comparisons with the dppm-bridged species $\mathbf{1 b}$.

Because of longer carbon chains between the phosphorus atoms, the intramolecular separations between the two $\left[\mathrm{Pt}\left(\mathrm{L}^{1}\right)\right]$ units in $\mathbf{1 0}$ and $\mathbf{1 1}$ are expected to be greater than in $\mathbf{1 b}$. This is reflected in their absorption spectra, which closely resembles that of the mononuclear phosphine congener $\left[\mathrm{Pt}\left(\mathrm{L}^{1}\right) \mathrm{PPh}_{3}\right]^{+}(\mathbf{8})$. No moderately intense absorption is evident above $400 \mathrm{~nm}$ like the ${ }^{1}\left[\mathrm{~d} \sigma^{*} \rightarrow \pi^{*}\right]$ absorption band in $\mathbf{1 b}$; hence, the $\left[\mathrm{Pt}\left(\mathrm{L}^{1}\right)\right]$ fragments in $\mathbf{1 0}$ and $\mathbf{1 1}$ are proposed to behave as discrete noninteracting moieties from a spectroscopic viewpoint. In contrast, the absorption spectrum of complex $\mathbf{9}$ in acetonitrile at room temperature displays a moderately intense band at $409 \mathrm{~nm}(\epsilon$ $=2500 \mathrm{dm}^{3} \mathrm{~mol}^{-1} \mathrm{~cm}^{-1}$ ). This low-energy band is absent in complex 10 and the mononuclear pyridine derivative $\left[\mathrm{Pt}\left(\mathrm{L}^{1}\right)\right.$ $\mathrm{py}^{+}(\mathbf{7})$, but the intensity of its tail $(>500 \mathrm{~nm})$ is noticeably

Table 7. Emission Data for Complexes 1b-6b and 9-11 in Acetonitrile (Complex Concentration $5 \times 10^{-5} \mathrm{M}$ )

\begin{tabular}{|c|c|c|}
\hline complex & $298 \mathrm{~K}$ data: $\lambda_{\max } / \mathrm{nm} ; \tau_{\mathrm{o}} / \mu \mathrm{s} ; \phi_{\mathrm{o}}$ & $77 \mathrm{~K}$ data: $\lambda_{\max } / \mathrm{nm}$ \\
\hline$\left[\mathrm{Pt}_{2}\left(\mathrm{~L}^{1}\right)_{2}(\mu\right.$-dppm $\left.)\right]\left(\mathrm{ClO}_{4}\right)_{2}, \mathbf{1 b}\left(\mathrm{ClO}_{4}\right)_{2}$ & $652 ; 0.14 ; 0.015$ & 638 \\
\hline$\left[\mathrm{Pt}_{2}\left(\mathrm{~L}^{2}\right)_{2}(\mu\right.$-dppm $\left.)\right]\left(\mathrm{ClO}_{4}\right)_{2}, \mathbf{2 b}\left(\mathrm{ClO}_{4}\right)_{2}$ & $659 ; 0.23 ; 0.016$ & 641 \\
\hline$\left[\mathrm{Pt}_{2}\left(\mathrm{~L}^{3}\right)_{2}(\mu\right.$-dppm $\left.)\right]\left(\mathrm{ClO}_{4}\right)_{2}, \mathbf{3} \mathbf{b}\left(\mathrm{ClO}_{4}\right)_{2}$ & $661 ; 0.19 ; 0.009$ & 644 \\
\hline$\left[\mathrm{Pt}_{2}\left(\mathrm{~L}^{4}\right)_{2}(\mu\right.$-dppm $\left.)\right]\left(\mathrm{ClO}_{4}\right)_{2}, \mathbf{4 b}\left(\mathrm{ClO}_{4}\right)_{2}$ & $654 ; 0.29 ; 0.024$ & 633 \\
\hline$\left[\mathrm{Pt}_{2}\left(\mathrm{~L}^{5}\right)_{2}(\mu-\mathrm{dppm})\right]\left(\mathrm{ClO}_{4}\right)_{2}, \mathbf{5 b}\left(\mathrm{ClO}_{4}\right)_{2}$ & $655 ; 0.40 ; 0.025$ & 640 \\
\hline$\left[\mathrm{Pt}_{2}\left(\mathrm{~L}^{6}\right)_{2}(\mu-\mathrm{dppm})\right]\left(\mathrm{ClO}_{4}\right)_{2}, \mathbf{6} \mathbf{b}\left(\mathrm{ClO}_{4}\right)_{2}$ & $662 ; 0.60 ; 0.018$ & 644 \\
\hline$\left[\mathrm{Pt}_{2}\left(\mathrm{~L}^{1}\right)_{2}(\mu-\mathrm{pz})\right]\left(\mathrm{ClO}_{4}\right), \mathbf{9}\left(\mathrm{ClO}_{4}\right)$ & $548 ; 0.2 ; 0.003$ & $555(\max ), 590,651$ \\
\hline$\left[\mathrm{Pt}_{2}\left(\mathrm{~L}^{1}\right)_{2}\left(\mu-\mathrm{dppC}_{3}\right)\right]\left(\mathrm{ClO}_{4}\right)_{2}, \mathbf{1 0}\left(\mathrm{ClO}_{4}\right)_{2}$ & $547 ; 1.9 ; 0.048$ & $530(\max ), 561$ \\
\hline$\left[\mathrm{Pt}_{2}\left(\mathrm{~L}^{1}\right)_{2}\left(\mu-\mathrm{dppC}_{5}\right)\right]\left(\mathrm{ClO}_{4}\right)_{2}, \mathbf{1 1}\left(\mathrm{ClO}_{4}\right)_{2}$ & $544 ; 0.8 ; 0.020$ & $532(\max ), 565$ \\
\hline
\end{tabular}


Table 8. Solvent Dependence of the ${ }^{3}\left[\mathrm{~d} \sigma^{*}, \pi^{*}\right]$ Emission of $\mathbf{1 b}\left(\mathrm{ClO}_{4}\right)_{2}$ and $\mathbf{5 b}\left(\mathrm{ClO}_{4}\right)_{2}$

\begin{tabular}{llc}
\hline & \multicolumn{2}{c}{$\lambda_{\max } / \mathrm{nm} ; \tau_{\mathrm{o}} / \mu \mathrm{s} ; \phi_{\mathrm{o}}$} \\
\cline { 2 - 3 } \multicolumn{1}{c}{ solvent } & \multicolumn{1}{c}{$\mathbf{1 b}$} & $\mathbf{5 b}$ \\
\hline dichloromethane & $640 ; 2.60 ; 0.18$ & $645 ; 2.45 ; 0.20$ \\
chloroform & $643 ; 2.45 ; 0.17$ & $647 ; 2.10 ; 0.19$ \\
acetone & $657 ; 0.49 ; 0.026$ & $657 ; 0.85 ; 0.051$ \\
acetonitrile & $652 ; 0.14 ; 0.015$ & $655 ; 0.40 ; 0.025$ \\
methanol & $654 ; 0.2 ; \phi_{\mathrm{o}}<0.01$ & $653 ; 0.30 ; \phi_{\mathrm{o}}<0.01$ \\
dimethylformamide & non-emissive & $675 ; 0.30 ; \phi_{\mathrm{o}}<0.01$
\end{tabular}

Table 9. Solid-State Emission Data for Complexes $\mathbf{1 b}-\mathbf{6 b}$ and 9-11

\begin{tabular}{|c|c|c|}
\hline complex & $\begin{array}{c}298 \mathrm{~K} \text { data: } \\
\lambda_{\max } / \mathrm{nm}\left(\tau_{\mathrm{o}} / \mu \mathrm{s}\right)\end{array}$ & $\begin{array}{c}77 \mathrm{~K} \text { data: } \\
\lambda_{\max } / \mathrm{nm}\end{array}$ \\
\hline$\left[\mathrm{Pt}_{2}\left(\mathrm{~L}^{1}\right)_{2}(\mu-\mathrm{dppm})\right]\left(\mathrm{ClO}_{4}\right.$ & $630(1.6)$ & 640 \\
\hline$\left[\mathrm{Pt}_{2}\left(\mathrm{~L}^{2}\right)_{2}(\mu\right.$-dppm $\left.)\right]\left(\mathrm{ClO}_{4}\right)_{2}, \mathbf{2} \mathbf{b}\left(\mathrm{ClO}_{4}\right)_{2}$ & 647( & 656 \\
\hline$\left[\mathrm{Pt}_{2}\left(\mathrm{~L}^{3}\right)_{2}(\mu\right.$-dppm $\left.)\right]\left(\mathrm{ClO}_{4}\right)_{2}, \mathbf{3 b}\left(\mathrm{ClO}_{4}\right)_{2}$ & 643( & 655 \\
\hline$\left[\mathrm{Pt}_{2}\left(\mathrm{~L}^{4}\right)_{2}(\mu\right.$-dppm $\left.)\right]\left(\mathrm{ClO}_{4}\right)_{2}, \mathbf{4 b}\left(\mathrm{ClO}_{4}\right)_{2}$ & $665(1.5)$ & 668 \\
\hline$\left[\mathrm{Pt}_{2}\left(\mathrm{~L}^{5}\right)_{2}(\mu\right.$-dppm $\left.)\right]\left(\mathrm{ClO}_{4}\right)_{2}, \mathbf{5 b}\left(\mathrm{ClO}_{4}\right)_{2}$ & $643(1.4)$ & 650 \\
\hline $\mathbf{6} \mathbf{b}\left(\mathrm{ClO}_{4}\right)_{2}$ & $670(1.7)$ & 674 \\
\hline$\left[\mathrm{Pt}_{2}\left(\mathrm{~L}^{1}\right)_{2}(\mu-\mathrm{pz})\right]\left(\mathrm{ClO}_{4}\right), \mathbf{9}\left(\mathrm{ClO}_{4}\right)$ & $\begin{array}{c}596(\max ) \\
620(1.0)\end{array}$ & $\begin{array}{r}580(\max ), \\
624,685\end{array}$ \\
\hline$\left[\mathrm{Pt}_{2}(\right.$ & 577 & 547,573 \\
\hline$\left.\left[\mathrm{Pt}_{2}\left(\mathrm{~L}^{1}\right)_{2}(\mu-\mathrm{dppC})_{5}\right)\right]\left(\mathrm{ClO}_{4}\right)_{2}, \mathbf{1 1}\left(\mathrm{ClO}_{4}\right)_{2}$ & 579 & 541,572 \\
\hline
\end{tabular}

smaller than that for complex $\mathbf{1 b}$ in the same spectral region (Figure 9). For assignment purposes, this absorption band exhibits characteristics which are transitional between ${ }^{1}$ MLCT and ${ }^{1}\left[\mathrm{~d} \sigma^{*} \rightarrow \pi^{*}\right]$. This is in accordance with the relatively long $\mathrm{Pt}-\mathrm{Pt}$ distance in 9 (3.612(2) $\AA$ ), which implies virtually no metal-metal interaction, although the presence of intramolecular $\pi-\pi$ contacts is feasible.

The luminescence properties of $\mathbf{9 - 1 1}$ in acetonitrile solution have been examined. Their respective emission energy is independent of complex concentrations from $10^{-6}$ to $10^{-3} \mathrm{M}$. The emission spectra of $\mathbf{1 0}$ and $\mathbf{1 1}$ in acetonitrile at room temperature show a structureless band centered at ca. $545 \mathrm{~nm}$. At $77 \mathrm{~K}$, the emissions blue-shift in energy and become vibronically resolved with spacing of ca. $1100 \mathrm{~cm}^{-1}$. This emissive behavior is similar to that for $\mathbf{8}$ and is evidently different from the ${ }^{3}\left[\mathrm{~d} \sigma^{*}, \pi^{*}\right]$ excited state of $\mathbf{1 b}$, which emits at lower energies without vibronic character. The solution emissions of $\mathbf{1 0}$ and $\mathbf{1 1}$ are assigned as ${ }^{3} \mathrm{MLCT}$ in nature.

The pyrazolate-bridged complex 9 emits in acetonitrile at room temperature with a peak maximum at $548 \mathrm{~nm}$. This structureless emission is similar to the ${ }^{3} \mathrm{MLCT}$ emissions of $\mathbf{1 0}$ and the mononuclear species 7 , but the $77 \mathrm{~K}$ frozen acetonitrile emission is strikingly different (Figure 10). A vibronically structured band is evident with the most intense peak at 555 $\mathrm{nm}$ and shoulders at 590 and $651 \mathrm{~nm}$. The room-temperature emission of a microcrystalline sample of $\mathbf{9}$ at room temperature is diffuse, but at $77 \mathrm{~K}$, a well-resolved vibronic structure (peaks at 580,624, and $685 \mathrm{~nm}$ ) is observed. While 9 displays negligible $\mathrm{Pt}-\mathrm{Pt}$ contacts at room temperature (as indicated by the crystal structure), the intramolecular separation between [Pt$\left(\mathrm{L}^{1}\right)$ ] units may decrease at $77 \mathrm{~K}$ to afford weak $\pi-\pi$ interactions. These would give rise to the low-energy shoulders in the solution- and solid-state emissions.

It is interesting to compare the solid-state emission spectra of $\left[\mathrm{Pt}_{2}(\operatorname{tpy})_{2}\left(\mu-\mathrm{L}^{\prime}\right)\right]^{3+}\left(\mathrm{L}^{\prime}=\right.$ anionic $\mathrm{N}-\mathrm{N}$ bridging ligand $)$ at $298 \mathrm{~K} \cdot{ }^{24}$ For example, the emission maxima for $\mathrm{L}^{\prime}=$ azaindolate $(\mathrm{Pt}-\mathrm{Pt}=3.13(2) \AA)$ and pyrazolate $(\mathrm{Pt}-\mathrm{Pt}=3.432(1) \AA)$ appear at 690 and $630 \mathrm{~nm}$, respectively; hence, a blue shift is evident with greater $\mathrm{Pt}-\mathrm{Pt}$ separations. In our study on $\left[\mathrm{Pt}_{2}-\right.$

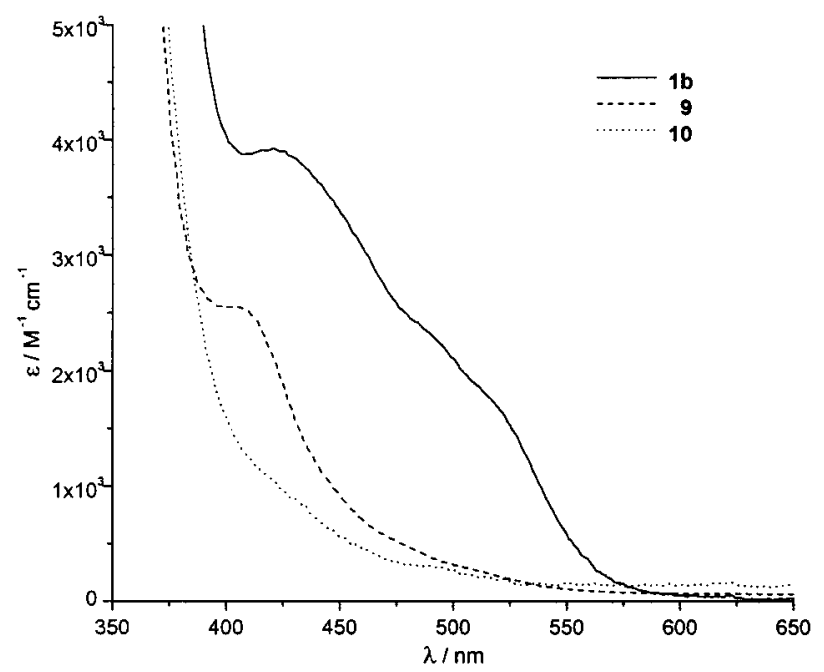

Figure 9. Lowest energy UV-vis absorption bands of $\mathbf{1 b}, \mathbf{9}$, and $\mathbf{1 0}$ in acetonitrile at $298 \mathrm{~K}$.

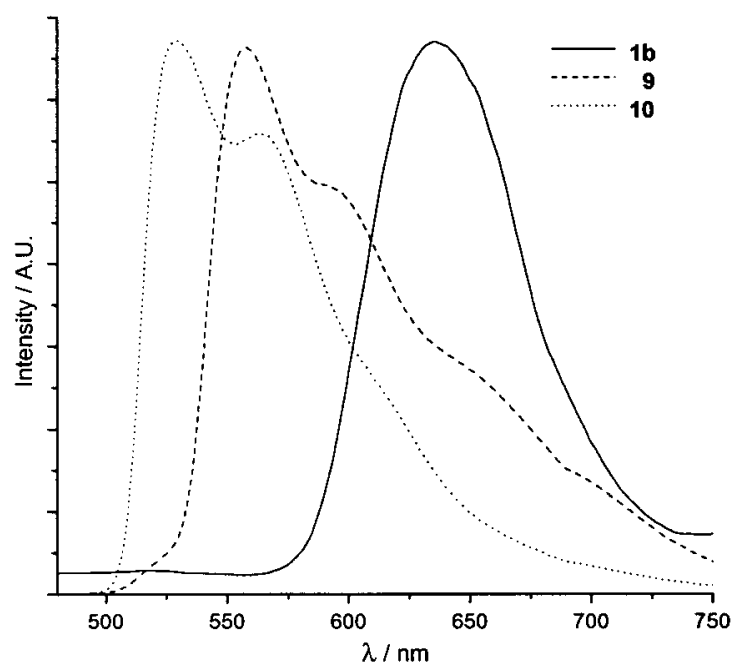

Figure 10. Normalized emission spectra of $\mathbf{1 b}, \mathbf{9}$, and $\mathbf{1 0}$ in acetonitrile at $77 \mathrm{~K}\left(\lambda_{\mathrm{ex}} 350 \mathrm{~nm}\right)$.

$\left.\left(\mathrm{L}^{1}\right)_{2}(\mu-\mathrm{L})\right]^{n+}$, the $298 \mathrm{~K}$ solid-state emission maxima for $\mathrm{L}=$ $\operatorname{dppm}(\mathrm{Pt}-\mathrm{Pt}=3.270(1) \AA)$ and pyrazolate $(\mathrm{Pt}-\mathrm{Pt}=3.612(2)$ $\AA$ ) appear at 630 and $596 \mathrm{~nm}$, respectively. It is apparent that as the $\left(\mathrm{L}^{1}\right) \mathrm{Pt}-\mathrm{Pt}\left(\mathrm{L}^{1}\right)$ separation is increased by lengthening $\mathrm{L}$, the origin of the excited state changes from ${ }^{3}\left[\mathrm{~d} \sigma^{*}, \pi^{*}\right]$ to ${ }^{3}$ MLCT and the solid-state emission is displayed at higher energies $(<600 \mathrm{~nm})$. From its transitional luminescent behavior, the $\mu$-pyrazolate derivative $\mathbf{9}$ apparently approaches the limit for metal-metal interaction.

Photoredox Properties of $\left[\mathrm{Pt}_{2}\left(\mathrm{~L}^{1}\right)_{2}(\mu\right.$-dppm) $]\left(\mathrm{ClO}_{4}\right)_{2}(\mathbf{1 b})$. The electro- and photochemical properties of $\mathbf{1 b}$ have been examined. The cyclic voltammogram of $\mathbf{1 b}$ reveals two quasireversible one-electron reduction waves $\left[\left(i_{\mathrm{pc}} / i_{\mathrm{pa}}\right) \approx 1\right]$ at -0.54 and $-0.77 \mathrm{~V}$ vs NHE with $0.1 \mathrm{M}^{\mathrm{n}} \mathrm{Bu}_{4} \mathrm{NPF}_{6}$ in acetonitrile (eqs 2 and 3 , respectively). The potentials are independent of scan

$$
\begin{gathered}
\mathrm{Pt}_{2}{ }^{2+}+\mathrm{e}^{-} \rightarrow \mathrm{Pt}_{2}{ }^{+} \quad E\left(\mathrm{Pt}_{2}{ }^{2+} / \mathrm{Pt}_{2}{ }^{+}\right)=-0.54 \mathrm{~V} \text { vs NHE } \\
\mathrm{Pt}_{2}{ }^{+}+\mathrm{e}^{-} \rightarrow \mathrm{Pt}_{2} \quad E\left(\mathrm{Pt}_{2}{ }^{+} / \mathrm{Pt}_{2}\right)=-0.77 \mathrm{~V} \text { vs NHE } \\
E\left({ }^{*} \mathrm{Pt}_{2}{ }^{2+} / \mathrm{Pt}_{2}{ }^{+}\right)=E\left(\mathrm{Pt}_{2}{ }^{2+} / \mathrm{Pt}_{2}{ }^{+}\right)+E_{0-0}
\end{gathered}
$$

rates from 20 to $100 \mathrm{mV} / \mathrm{s}$. The excited-state potential can be estimated by eq $4\left(\mathrm{Pt}_{2}=\left[\mathrm{Pt}_{2}\left(\mathrm{~L}^{1}\right)_{2}(\mu\right.\right.$-dppm) $\left.)\right]$. 
Table 10. Rate Constants for Reductive Quenching of the ${ }^{3}\left[\mathrm{~d} \sigma^{*}, \pi^{*}\right]$ Emission of $\mathbf{1 b}\left(\mathrm{Pt}_{2}{ }^{2+}\right)$ by Organic Donors in Dichloromethane at $20 \pm$ $0.2{ }^{\circ} \mathrm{C}$

\begin{tabular}{|c|c|c|c|c|}
\hline organic quencher & $E\left(\mathrm{D}^{+} / \mathrm{D}^{\circ}\right)^{a} / \mathrm{V}$ & $k_{\mathrm{q}} / \mathrm{dm}^{3} \mathrm{~mol}^{-1} \mathrm{~s}^{-1}$ & $k_{\mathrm{q}}{ }^{\prime} / \mathrm{dm}^{3} \mathrm{~mol}^{-1} \mathrm{~s}^{-1}$ & $\ln k_{\mathrm{q}}{ }^{b}$ \\
\hline$N, N, N^{\prime}, N^{\prime}$-tetramethyl-p-phenylenediamine & 0.35 & $1.08 \times 10^{10}$ & $2.35 \times 10^{10}$ & 23.88 \\
\hline$N, N, N^{\prime}, N^{\prime}$-tetramethylbenzidine & 0.67 & $1.16 \times 10^{10}$ & $2.76 \times 10^{10}$ & 24.04 \\
\hline$o$-phenylenediamine & 0.75 & $4.80 \times 10^{9}$ & $6.32 \times 10^{9}$ & 22.57 \\
\hline benzidine & 0.79 & $6.21 \times 10^{9}$ & $9.01 \times 10^{9}$ & 22.92 \\
\hline phenothiazine & 0.86 & $6.65 \times 10^{9}$ & $9.96 \times 10^{9}$ & 23.02 \\
\hline diethylaniline & 0.94 & $4.61 \times 10^{9}$ & $5.99 \times 10^{9}$ & 22.51 \\
\hline diphenylamine & 1.07 & $1.24 \times 10^{9}$ & $1.31 \times 10^{9}$ & 21.00 \\
\hline$p$-chloroaniline & 1.31 & $6.18 \times 10^{8}$ & $6.38 \times 10^{8}$ & 20.27 \\
\hline 2,4-dichloroaniline & 1.46 & $6.02 \times 10^{6}$ & $6.02 \times 10^{6}$ & 15.61 \\
\hline 1,4-dimethoxybenzene & 1.58 & $1.20 \times 10^{6}$ & $1.20 \times 10^{6}$ & 14.00 \\
\hline 1,2,3-trimethoxybenzene & 1.66 & $2.06 \times 10^{5}$ & $2.06 \times 10^{5}$ & 12.24 \\
\hline
\end{tabular}

The $0-0$ transition energy of $\mathrm{Pt}_{2}{ }^{2+}, E_{0-0}$, can be estimated from the overlap of the emission and excitation spectra, which occurs at $2.15 \mathrm{eV}$. Thus $* \mathrm{Pt}_{2}{ }^{2+}$ is a strong oxidant with $E\left(* \mathrm{Pt}_{2}{ }^{2+} /\right.$ $\left.\mathrm{Pt}_{2}{ }^{+}\right)$at $1.61 \mathrm{~V}$ vs NHE. The excited-state redox potential has also been estimated through quenching studies with a series of aromatic hydrocarbons in dichloromethane. The chosen aromatic hydrocarbons have similar size and electronic structure but different reduction potentials $E\left(\mathrm{D}^{+} / \mathrm{D}^{\circ}\right)$. For each quencher, the Stern-Volmer plot is linear over the range of quencher concentrations. Analysis of the quenching rate data was performed by the method of Meyer and co-workers. ${ }^{38}$ Quenching rate constants and the reduction potentials of the quenchers are summarized in Table 10. From the plot of $\ln k_{\mathrm{q}}{ }^{\prime} \mathrm{vs} E\left(\mathrm{D}^{+} / \mathrm{D}^{\circ}\right)$ $(\mathrm{D}=$ quencher; see Supporting Information), the excited-state potential $E\left({ }^{*} \mathrm{Pt}_{2}{ }^{2+} / \mathrm{Pt}_{2}{ }^{+}\right)$is estimated to be $1.63 \mathrm{~V}$ vs NHE, which is in close agreement with the value of $1.61 \mathrm{~V}$ calculated from the spectroscopic and electrochemical data.

\section{Concluding Remarks}

The mono- and binuclear complexes reported in this study exhibit luminescence with relatively large quantum yields in solution at room temperature. We have shown that cyclometalating tridentate ligands based on 6-phenyl-2,2'-bipyridine offer favorable photophysical characteristics compared to $2,2^{\prime}: 6^{\prime}, 2^{\prime \prime}$ terpyridine in square planar platinum(II) systems. Because of the ease in modifying the bridging ligands, these complexes represent a new class of luminophores with potentially tunable

(38) For details of data treatment, see: Bock, C. R.; Connor, J. A.; Gutierrez, A. R.; Meyer, T. J.; Whitten, D. G.; Sullivan, B. P.; Nagle, J. K. J. Am. Chem. Soc. 1979, 101, 4815, and references therein. excited-state properties. The planar geometry of the mononuclear derivatives facilitates face-to-face interactions, and self-quenching of the emissions have been observed at room temperature in dichloromethane.

On the basis of the structural parameters and spectroscopic assignments described in this work, it is proposed that metalmetal interactions in this system will yield ${ }^{3}\left[\mathrm{~d} \sigma^{*}, \pi^{*}\right]$ excited states which emit above $600 \mathrm{~nm}$. As the $\mathrm{Pt}-\mathrm{Pt}$ separation lengthens, the energy of the $\left[\mathrm{d} \sigma^{*}, \pi^{*}\right]$ excited state increases and the nature of the excited state accordingly switches to MLCT with emissions below $600 \mathrm{~nm}$. The transitional behavior of $\left[\mathrm{Pt}_{2}\left(\mathrm{~L}^{1}\right)_{2}(\mu-\mathrm{pz})\right]^{+}$, from structural and photophysical perspectives, is noteworthy and provides a "reference point" for future investigations into $\mathrm{d}^{8}-\mathrm{d}^{8}$ interactions. Hence a correlation between (1) the energy of the emission band, (2) the nature of excited state, and (3) the degree of metal-metal interaction is established.

Acknowledgments. We are grateful for financial support from The University of Hong Kong. The work described in this paper was partially supported by a grant from the Research Grants Council of the Hong Kong Special Administrative Region, China [HKU 7298/99P].

Supporting Information Available: Listings of crystal data, atomic coordinates, calculated coordinates, anisotropic displacement parameters, and bond lengths and angles for $4 \mathbf{b}\left(\mathrm{ClO}_{4}\right)_{2} \bullet 5 \mathrm{H}_{2} \mathrm{O}$ and $\mathbf{9}\left(\mathrm{PF}_{6}\right)$, excitation spectrum of $\mathbf{5 b}$ in acetonitrile at $77 \mathrm{~K}$, and a plot of $\ln k_{\mathrm{q}}{ }^{\prime} \mathrm{vs}$ $E\left(\mathrm{D}^{+} / \mathrm{D}^{\circ}\right)$. This material is available free of charge via the Internet at http://pubs.acs.org.

IC990238S 